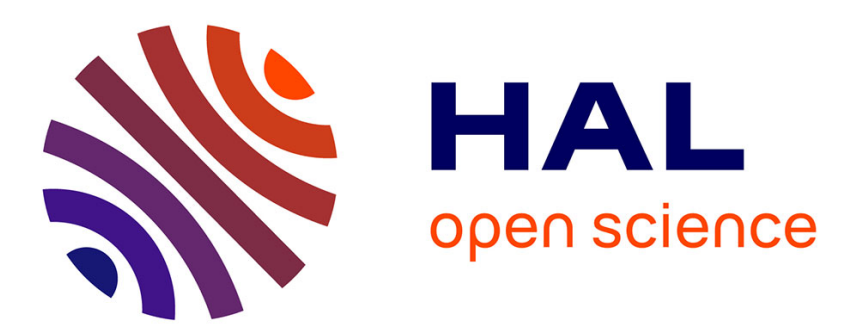

\title{
Toward local identification of cohesive zone models using digital image correlation
}

Vincent Richefeu, André Chrysochoos, Vincent Huon, Yann Monerie, Robert Peyroux, Bertrand Wattrisse

\section{- To cite this version:}

Vincent Richefeu, André Chrysochoos, Vincent Huon, Yann Monerie, Robert Peyroux, et al.. Toward local identification of cohesive zone models using digital image correlation. European Journal of Mechanics - A/Solids, 2012, 34, pp.38-51. 10.1016/j.euromechsol.2011.12.001 . hal-00832307

\section{HAL Id: hal-00832307 https://hal.science/hal-00832307}

Submitted on 10 Jun 2013

HAL is a multi-disciplinary open access archive for the deposit and dissemination of scientific research documents, whether they are published or not. The documents may come from teaching and research institutions in France or abroad, or from public or private research centers.
L'archive ouverte pluridisciplinaire HAL, est destinée au dépôt et à la diffusion de documents scientifiques de niveau recherche, publiés ou non, émanant des établissements d'enseignement et de recherche français ou étrangers, des laboratoires publics ou privés. 


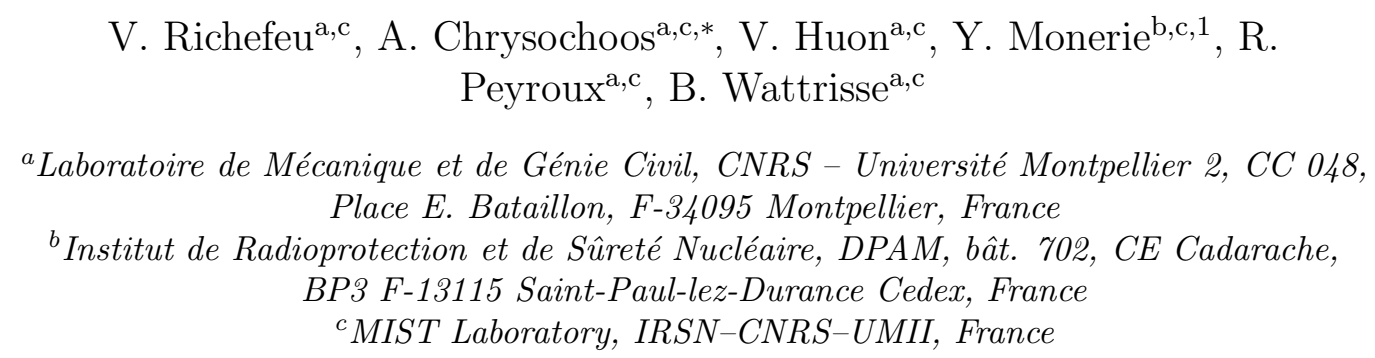

10

\section{Abstract}

In recent years, cohesive-zone models have been formulated and used to numerically simulate the fracture of solid materials. Cohesive-zone models presented in the literature involve a 'jump' in the displacement field describing crack onset within a predefined interface network corresponding to interfaces between elements of the finite element (FE) mesh. The introduction of a virtual displacement jump is convenient to numerically manage micro-crack or void initiation, growth and coalescence. Until now, the forms of interface laws were mainly chosen semi-empirically in connection with the overall responses of specimens when subjected to standard loadings. In this study, a cohesive-zone model identification method is proposed based on the local material behavior derived from kinematical measurements obtained by digital image correlation (DIC). A series of tensile loadings were performed for several damageable elastic-plastic materials on standard tensile specimens. Kinematical data analysis enabled early detection and tracking of the zone where the crack will finally occur. The results of this study highlight the

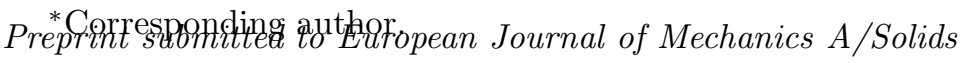

October 14, 2011 
potential of DIC to quantify damage and show how damage assessments can be inserted in cohesive-zone model identification.

\section{Introduction}

Cohesive-zone models (CZMs), which were first introduced through the pioneering work of Dugdale (1960) and Barenblatt (1962), are suitable for simulating fractures in a wide range of materials and to account for heterogeneities at various scales from the grain to the structure (see e.g. the review of de Borst et al. (2006)). In the so-called 'cohesive-volumetric finite element' framework, CZMs are introduced at interfaces between adjacent elements of a finite element discretization (see Figure 1). They have been successfully used to simulate and predict the entire fracture process from crack onset to rupture, including crack growth, propagation, potential bifurcation, multiple fracturing, etc. (see e.g. Perales et al. (2010) for a review and references therein).

In recent years, substantial progress has been achieved from a numerical standpoint, along with regular improvement of CZM predictions. In particular, studies have been carried out to assess the impact of the shape of CZMs (bilinear, exponential, trapezoidal, etc.). A range of information can be extracted from recent literature. Chandra et al. (2002) and Tvergaard and Hutchinson (1992) found that the shape only had a slight influence on numerical predictions, with the main parameters being the ultimate stress and fracture energy. On the contrary, Alfano (2006) noted that the numeri- 


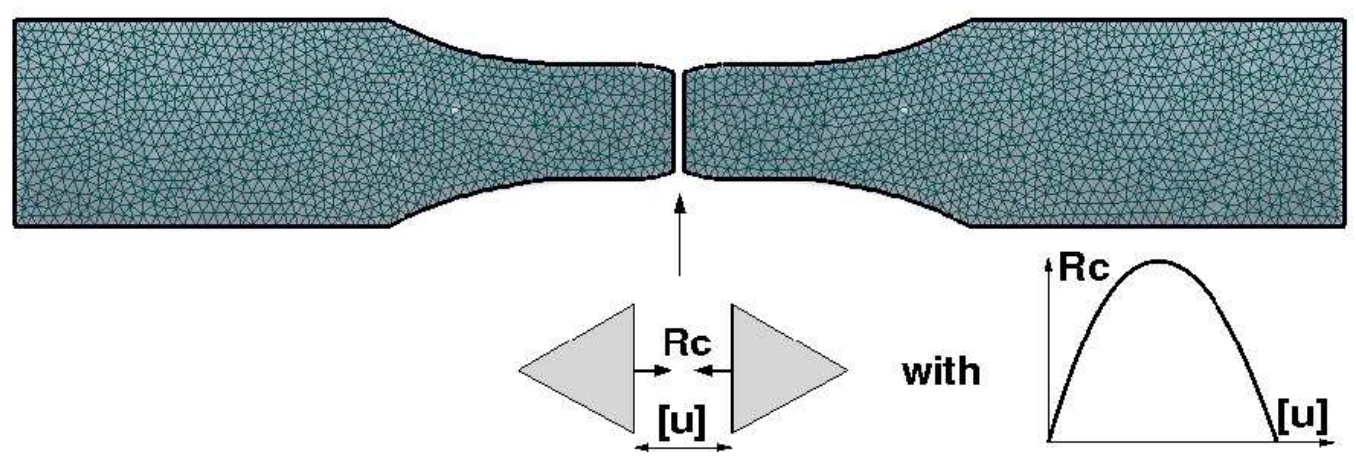

Figure 1: Sketch of a sample after a uniaxial traction test using the cohesive/volumetric finite element approach. Cohesive zone models are embedded along connected meshes and describe the traction-separation relationship as a softening curve. When the traction vanishes the cohesive bond is broken and the crack occurs (the resulting crack path can be complex; this figure exhibits a straight crack for sake of simplicity).

cal behavior of a metal matrix/composite was very sensitive to the shape of the interfacial constitutive relation of the CZM and the review of de Borst et al. (2006) underlines that the shape has to be chosen on the basis of an empirical rule that is popular throughout the numerical research commu37 nity: 'triangle shapes' are convenient for modeling brittle materials, while ${ }_{38}$ 'door-like shapes' are suitable for ductile materials. Moreover, Kubair and Geubelle (2003) show that the stability of cohesive models due to the surface softening behavior depends on the shape of the model: extrinsic CZMs

${ }_{41}$ (models with infinite initial stiffness, i.e. the cohesive traction is equal to the 42 material strength) are more stable than intrinsic ones (models with finite ini43 tial stiffness, i.e. the traction-separation relationship exhibits a finite slope). 44 Concerning these intrinsic models, Chaboche et al. (2001); Perales et al. 45 (2010) Tomar et al. (2004) underline the influence of an additional surface 46 compliance on the overall response of a fully cohesive-volumetric formulation. 
Although the CZM technique is becoming increasingly robust, its application is somewhat arbitrary and there are still several difficulties, notably regarding the identification of constitutive equations. It seems that, in addition to the mechanical parameters of CZMs, the shape also has to be determined.

Some recent attempts have been proposed in the literature to derive cohesive laws from experimental measurements. At the nanoscale, these attempts often concern the identification of a potential from which the cohesive model derived (Jiang, 2010; Ngo et al., 2010). At higher scales, most studies have been based on the assumption of a general shape for the constitutive equations and a predefined crack path, and the material parameters have been derived from experimental strain data (see e.g. Andena et al. (2006) among others for pure mode I conditions). Hong and Kim (2003) propose an inverse identification of cohesive laws without any assumptions on the shape of the traction-separation relationship. This approach is based on some eigenfunction expansion of elastic far-fields surrounding a cohesive crack tip and a field projection method. This inverse method is, by construction, limited to elastic bulk behaviors but was successfully used by Arias et al. (2007) to validate cohesive-based simulations of dynamic fractures and was recently extended to elastic-plastic bulk behaviors through a hybrid numerical-experimental approach. Full experimental identification of cohesive laws without any assumptions on the bulk behavior or on the shape of the CZM was proposed by (Tan et al., 2005) using some digital image correlation (DIC) techniques. This identification was, however, limited to predefined crack paths. To our best of knowledge, all recent works dealing with CZM identification with DIC techniques are limited to: (a) an identification of the 
cohesive parameters, i.e. the shape of the cohesive law is a priori fully given (Fedele et al., 2009; Valoroso and Fedele, 2010) or partly given (Shen and Paulino, 2011), and/or (b) predefined crack paths, i.e. debond of interfaces (Valoroso and Fedele, 2010; Zhu et al., 2009) or precracked samples (Fuchs and Major, 2011). In conclusion, no direct experimental method is currently available for identifying cohesive laws - shape and parameters - in elastoplastic materials where a crack emerges naturally from the overall loading (no predefined crack path). The main questions addressed in this paper are thus the following.

1. Is there any experimental evidence of the cohesive-volumetric decomposition relevance, in particular for elatoplastic damageable materials?

2. If so, can the associated CZM be directly deduced from the surrounding bulk material behavior?

3. If so, can the shape and the mechanical parameters of the CZM be simultaneously identified even if the locus of the crack onset is not known a priori?

4. And, as a consequence of the previous question: is early detection of the locus of the main crack possible during a standard mechanical test such as uniaxial tension test on homogeneous materials without any precracks?

Here we propose answers to these questions based on experimental measurements and the proposed experimental methodology is founded on full-field measurement techniques which, as pointed out by Tan et al. (2005), seem promising to enhance choices of CZM constitutive equations and improve their identification. 
Over the last 10ten years, there has been substantial development in quantitative imaging techniques, with widespread applications in the mechanics of materials. Digital image correlation (DIC), for instance, has become a powerful technique that provides reliable kinematic measurement fields (displacement, and with the help of some numerical differentiation, strain, strain-rate, acceleration, etc.). DIC is a fully non-intrusive and sensitive measurement tool that can be used to monitor material surface displacements in a wide range of engineering materials such as metals, polymers, ceramics and concretes (Corr et al., 2007; Daly et al., 2007; Fang et al., 2006; Mekky and Nicholson, 2006).

As already announced, the goal of this paper is to propose a CZM identification based on full-field measurements (DIC). This identification does not assume neither any particular shape nor any predefined crack path, but focuses on the experimental validity of the projection of volumic (micro) damage onto a simple surface. Because of the difficulty of this task, the study is restricted to standard metallic materials subjected to overall uniaxial tension. In particular, the local effect of the stress triaxiality on the volumic damage is neglected and we propose a simple and pragmatic method to identify the normal part of the cohesive law (1D approach). The experiment methodology is composed of three steps.

First, the zone where a crack initiates and propagates in mode I is carefully identified. As previously shown in other situations, we underline that this zone corresponds to the strain localization locus, and can by early detected and tracked by image analysis (inflection point of the longitudinal velocity profile). Moreover, the 1D approach can be warranted by the kine- 
matical data which show very low elastoplastic rotations, even in the localization zone. This zone is, as expected, the region where necking occurs and damage preferentially develops.

Second, the kinematic data may also give fields of void fraction since isotropic straining of the specimen cross sections is supposed. A simple model of spherical voids is here employed, but any convenient micromechanical model can be used instead. This spherical voids model was selected for its simplicity and to illustrate the experimental protocol.

Third, the method proposes to construct a local stress-strain correspondence, which can be used to identify an elastoplastic damageable constitutive equation from which the cohesive law can be extracted. The correspondence is first explained and illustrated on two academic bulk behaviors: 1 / the damageable elasticity and $2 /$ the standard damageable elastoplasticity. From these academic examples, we derive the generality of the correspondence which thus can be employed without any assumption on the bulk behavior. The experimental uniaxial response of any damageable material is then converted onto a bulk behavior with only hardening (no damage) and a cohesive zone model incorporating all the softening effects. Such a cohesive law is hence suitable for describing damaging up to cracking. The shape of the identified CZMs changes with the material ductility and appears to be in close agreement with some standard CZM formulations used in the computational codes. 


\section{Experimental procedures}

\subsection{Experimental setup and correlation technique}

The experimental setup shown in Figure 2 involves a uniaxial testing machine equipped with a $100 \mathrm{kN}$ load cell and a CCD camera. Its optical axis is set perpendicular to the specimen surface and remains fixed during the test.

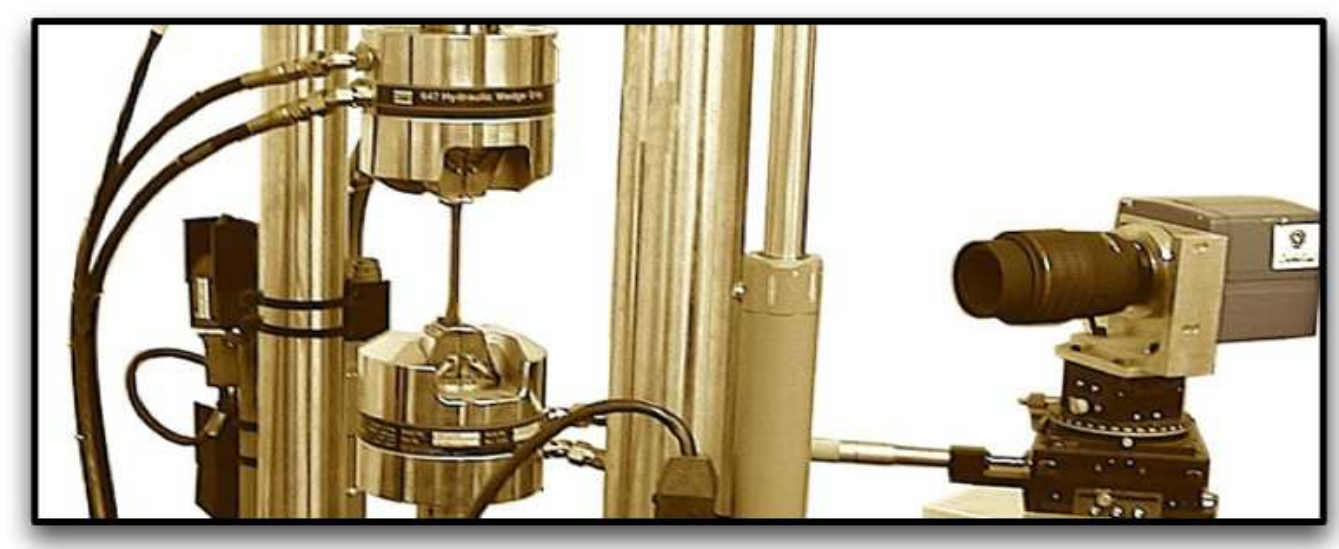

Figure 2: A general view of the experimental setup.

The speckle of the digitized image represents local optical signatures, which are used to track the material surface elements. The in-plane displacement vectors are obtained by a direct digital image correlation method. A normalized discrete correlation function is computed at selected pixels (e.g. initially positioned on a regular grid) of consecutive images. For this study, we used KelKins image processing software (Bornert et al., 2009) developed for the computation of kinematical fields. The surface of the specimen observed by the visible CCD camera is speckled with black and white paint in order to obtain a random pattern defining the local optical signature of each 
material surface element. A classical digital image correlation algorithm allowed us to determine the in-plane components of the displacement field on a regular rectangular grid. To determine the displacement at each point of the grid, we choose to perform a direct correlation computation and use a normalized intercorrelation function $\mathcal{C}(\phi)$ (see Latourte et al. (2008) for details):

$$
\mathcal{C}(\phi)=\left\langle I_{1}, I_{2}^{\phi}\right\rangle / \sqrt{\left\langle I_{1}, I_{1}\right\rangle\left\langle I_{2}^{\phi}, I_{2}^{\phi}\right\rangle}
$$

where $I_{1}$ and $I_{2}$ are the intensity functions of two images separated by a small strain increment, $I_{2}^{\phi}(\boldsymbol{x})=I_{2}(\boldsymbol{x}+\boldsymbol{\phi}(\boldsymbol{x}))$ for any point $\boldsymbol{x}$ and $\langle a, b\rangle$ is the scalar product of any light intensities $a$ and $b$ on the correlation subset $Z:\langle a, b\rangle=\int_{Z} a(x) \cdot b(x) d x$. With a zero order function $\phi$ and a computation of the intercorrelation function for multiple pixel shifts, the displacements are estimated with a 1-pixel resolution. To achieve higher resolutions, the discrete intercorrelation function is interpolated in the neighborhood of its discrete maximum by a quadratic polynomial (Wattrisse et al., 2001a). The in-plane velocity and strain components were derived from the displacement data by a numerical differentiation method based on a local polynomial approximation of the displacement field. Local time fitting of displacement fields often involves a second order polynomial while local space fitting is associated with a coupled first order polynomial of the two in-plane coordinates. Recent works show that in most situations the error on the displacement field is the combination of two contributions (Bornert et al., 2009): 1/ the model error related to the mismatch between the real transformation and one used as the shape function on the 
displacement field, and $2 /$ the ultimate error linked to the propagation of the image noise on the displacement and to the gray levels interpolation bias. In order to minimize both contributions in situations involving strongly localized strain fields, we chose to use an incremental strain computation (the correlation grid is regularly upgraded every $5 \%$ macroscopic deformation increments), and we used bi-linear shape functions on the displacement fields. The image processing has been widely presented in previous works (Wattrisse et al., 2001a). Readers interested in its application can also refer to Huon et al. (2007); Latourte et al. (2008); Wattrisse et al. (2001b,c) or Chrysochoos et al. (2008) where the image processing was applied to different types of materials, i.e. metals, shape memory alloys, polymers, ceramics, reinforced concretes. In the case of brittle materials (e.g. ceramics and concretes), natural speckle was advantageously used. Indeed the cracks that occur at the specimen surface may induce a degradation of the local optical signatures and bad image correlations can be performed. For ductile materials, recent work (Lopez-Crespo et al., 2009) showed that it is possible to still use natural speckle to analyse elastic-plastic behaviour around a crack tip. However, in the present application, the polished surface of specimens forced us to use black and ithwhite paint. To limit and check the possible correlation problems, an incremental image processing was done verifying at each computational step the quality of the correlation factor.

\subsection{Materials and test procedure}

Monotonic tensile tests were performed on flat specimens at room temperature (about $293 \mathrm{~K})$. A slow crosshead velocity $(2.5 \mathrm{~mm} / \mathrm{min})$ was kept 
Table 1: Measured properties of the tested materials: Young's modulus (E), tensile yield stress $\left(\sigma_{y}\right)$, maximal conventional stress $\left(\sigma^{\text {peak }}\right)$, (mean Hencky) failure strain $\left(\varepsilon_{11}^{\max }\right)$, and initial cross section $\left(S_{0}\right)$.

\begin{tabular}{lcccccc}
\hline Material & Abbr. & $\begin{array}{c}E \\
(\mathrm{GPa})\end{array}$ & $\begin{array}{c}\sigma_{y} \\
(\mathrm{MPa})\end{array}$ & $\begin{array}{c}\sigma^{\text {peak }} \\
(\mathrm{MPa})\end{array}$ & $\varepsilon_{11}^{\max }$ & $\begin{array}{c}S_{0} \\
(\mathrm{~mm} \times \mathrm{mm})\end{array}$ \\
\hline High-strength steel & Steel 1 & 150 & 360 & 630 & 0.22 & $13.5 \times 2.5$ \\
Ductile steel (Lüders bands) & Steel 2 & 210 & 380 & 450 & 0.31 & $10 \times 2.5$ \\
2024 T3 aluminium alloy & Dural & 75 & 310 & 480 & 0.15 & $10 \times 3$ \\
Copper (99\%) & $\mathrm{Cu}$ & 100 & 200 & 290 & 0.23 & $13.5 \times 2$ \\
\hline
\end{tabular}

constant throughout all tests. During the tensile test, 12-bit grey level images were captured at a frame rate of $2 \mathrm{~Hz}$. The optical lens used allowed us to obtain a spatial resolution of about $40 \mu \mathrm{m}$.pixel ${ }^{-1}$. Force signal $\mathcal{F}$, crosshead displacement $\Delta L$ and time $t$ were also recorded and stored in each acquired image file. As shown in Figure 3, subscripts 1, 2 and 3 respectively correspond to the length, width and depth of the specimen, with DIC giving the in-plane components $u_{1}$ and $u_{2}$ of the displacement during tensile loading in direction 1.

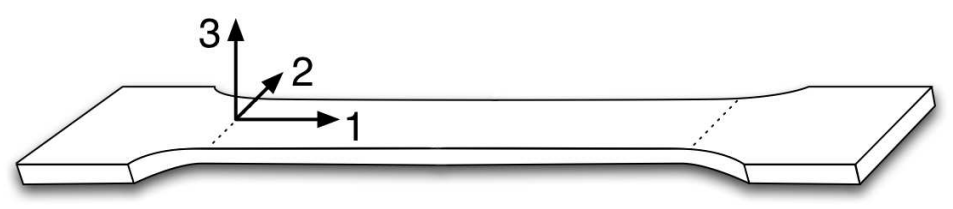

Figure 3: Schematic representation of the sample and frame of reference.

The main mechanical characteristics of the tested specimens are summarized in Table1. Figure 4a shows the conventional stress $\sigma_{\mathrm{c}}=\mathcal{F} / S_{0}$ (where $S_{0}$ is the initial cross section of the specimen gauge part) as a function of the 
mean Hencky strain $\bar{\varepsilon}_{11}$ (computed throughout the sample gauge part), in the loading direction for each specimen. These materials are ductile but show different modes of failure. In particular, Steel 1 and Dural specimens failed suddenly while the fracture of Steel 2 and $\mathrm{Cu}$ specimens was more progressive. Steel 2 presented a plastic plateau associated with the displacement throughout the specimen gauge part of a high strain rate zone associated with Lüders band propagation.
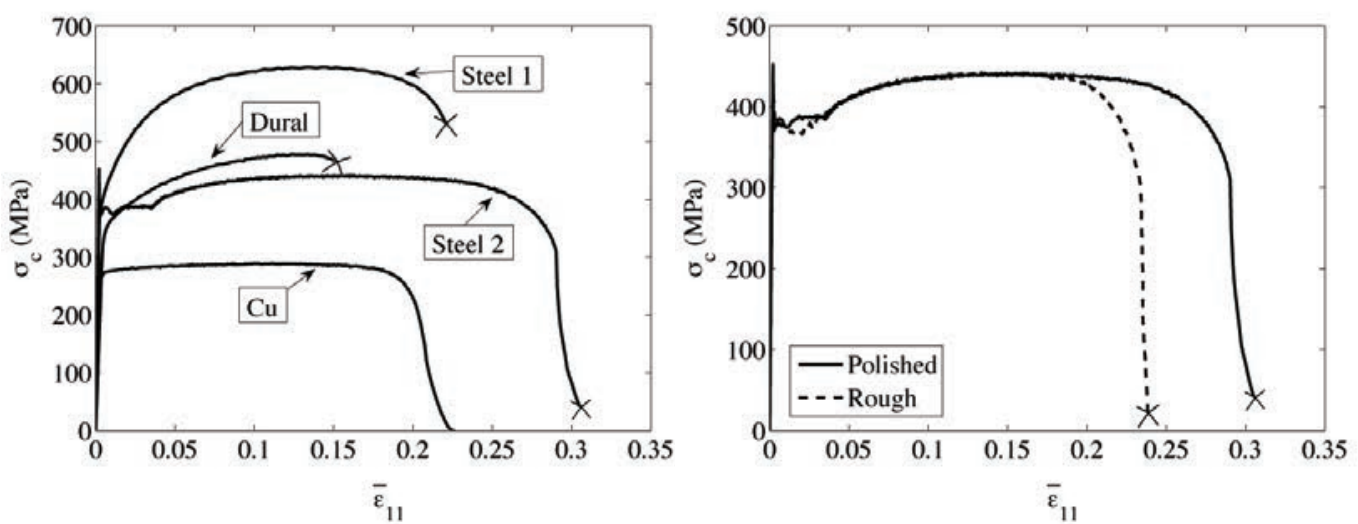

Figure 4: Uniaxial conventional stress $\sigma_{\mathrm{c}}$ vs. mean Hencky strain $\bar{\varepsilon}_{11}$ diagrams: (a) tested materials. (b) Example of polishing influence for the Steel 2 specimens. Crosses are associated with the specimen rupture.

Even slight geometrical imperfections of the specimen surface can give rise to early crack inception. We then performed comparative tensile tests on polished and unpolished specimens to highlight the influence of geometrical surface defects on the overall specimen response. Figure $4 \mathrm{~b}$ shows the two responses in terms of $\sigma_{\mathrm{c}}$ vs. $\bar{\varepsilon}_{11}$ diagrams. As expected, we observed no significant difference before the maximum load, but the curves diverged significantly during the specimen softening, i.e. greater strains were reached with polished specimens. As the onset of localization phenomena is crucial 
for CZM identification, the required tests were systematically performed with specimens polished on all faces.

\section{Experimental observations}

During tensile tests, the gauge part of the specimen is generally considered as a material volume uniformly stressed and strained. This convenient hypothesis allows the experimenterexperimentalist to estimate stress and strain from load cell and extensometer signals. However, several years ago, based on our concern about obtaining reliable full-field strain measurements, we began to question the homogeneity of these 'simple' tensile tests and to consider the gauge part of specimens as a possibly non-uniformly strained structure (Wattrisse et al., 2001b). The next sub-section shows some aspects of the early and gradual development of strain localization. We arbitrarily chose kinematical results obtained with $\mathrm{Cu}$ samples to illustrate the field properties that will be used to detect and assess damage in the sequel.

\subsection{Localization zone}

The early and gradual development of localization can be observed in Figure 5 which represents the temporal evolution of longitudinal Eulerian strain rate profiles $\mathrm{D}_{11}\left(X_{1}, X_{2}=0, t\right)$, where $X_{1}$ and $X_{2}$ denote the in-plane Lagrangian coordinates. This longitudinal profile, using contour plots, was captured at each time $t$ in the middle of the gauge part of the specimen (i.e. $\left.X_{2}=0\right)$. Contour plots were chosen to underline the progressive narrowing of the localization zone. The load signal was also superimposed to indicate the overall sample response. Note that the apparent slope of the loadtime curves (Figure 5 and the following) can be slightly different in 

strain rate on the specimen gauge length.

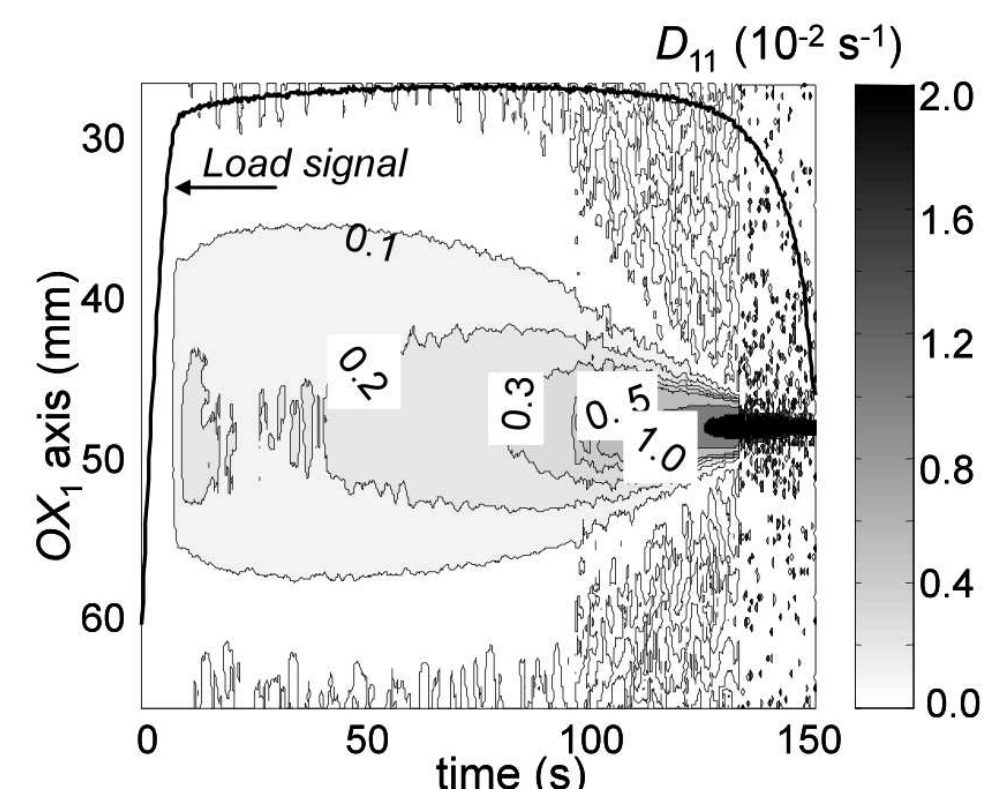

Figure 5: Copper specimen. Time course of the longitudinal components $\mathrm{D}_{11}\left(X_{1}, X_{2}=0\right.$, $t$ ) of the Eulerian strain rate tensor. Profiles were captured at $X_{2}=0$, i.e. along the $O X_{1}$ symmetry axis of the sample.

comparison to the slope of the load-strain curves (Figure 4) since the overall constant velocity can lead to a non constant average

Another illustration of the localization precocity can be given in terms of acceleration. Generally, for quasi-static loadings, the acceleration amplitude remains negligible as compared with the gravity acceleration, this latest being often ignored in equilibrium equations. Nevertheless, the high performances of current DIC techniques allow reliable assessments of very low acceleration intensity and the analysis of acceleration fields appears to be of some interest for early detection of material flow heterogeneity. Using the same graphical format as the one used in Figure 5, the longitudinal profile of the acceleration component $\gamma_{1}=\partial^{2} u_{1} / \partial t^{2}$, where $u_{1}$ is the longitudinal displacement, is plot- 
ted in Figure 6a. At the very beginning of the test, acceleration data were not plotted until a 'significant' signal was reached in order to avoid parasitic effects of the finite stiffness of the testing machine. At the end of the test, acceleration data were no longer plotted once the displacement gradients became too high for the spatial and temporal resolutions of the CCD camera, thus inducing an awkward fitting (inconsistent smoothing) of the acceleration data. The vertical dashed lines correspond to the profiles plotted in Figure $6 \mathrm{~b}$ while the horizontal dashed line (placed at $X_{1} \simeq 48 \mathrm{~mm}$ ) roughly separates regions with positive and negative accelerations. This line then corresponds to a specimen cross-section characterized by a zero-acceleration, or in other words to the center of the strain localization zone shown in Figure 5. This has already been observed in stroke-controlled tests (Wattrisse et al., 2001b), the strain localization zone corresponding to the inflection point of the longitudinal velocity profile. We then estimated that the damage preferentially developed in such zone where the crack finally occurs. Figure $6 \mathrm{~b}$ shows examples of longitudinal acceleration profiles $\gamma_{1}\left(X_{1}, X_{2}=0, t\right)$ captured at $t=40,80$ and $120 \mathrm{~s}$, respectively. Note that the reconstruction of acceleration profiles in the Lagrange configuration is necessary to make the profiles comparable. It can be verified that the encircled point located at $X_{1} \simeq 48 \mathrm{~mm}$ is the sole point keeping a zero-acceleration throughout this velocity-controlled test.

In Figure 7a-d (left), profiles of the longitudinal acceleration $\gamma_{1}\left(X_{1}, X_{2}=\right.$ $0, t)$ obtained for the 4 studied materials are shown. To facilitate the comparison of material responses we used normalized scales of time and space, with $t^{\star}=1$ corresponding to the instant of specimen breaking and $X_{1}^{\star}$ corre- 


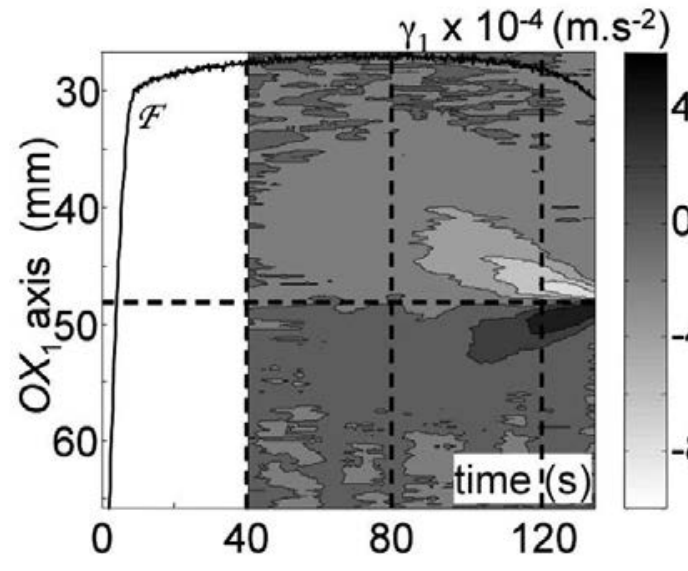

(a)

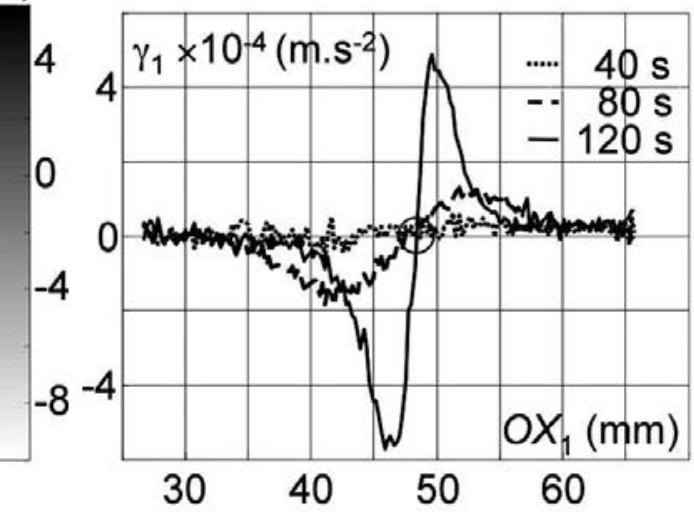

(b)

Figure 6: Copper specimen. (a) Lagrangian representation of the time course of the acceleration profile $\gamma_{1}\left(X_{1}, X_{2}=0, t\right)$. (b) Acceleration distributions along the $O X_{1}$ axis at 3 given times. The intersection point of the 3 profiles corresponds to the zeroacceleration point.

sponding to the initial gage length of the specimen. The conventional stress was as usual superimposed to give a landmark of the specimen response.

Except for Steel 2 (Figure 7b), where the passage of the Lüders band at the very beginning of strain hardening induced a deceleration-acceleration propagating wave, a steady concentration of level curves of positive and negative acceleration was clearly observed on both sides of the necking zones. The section where the fracture eventually took place was then characterized by zero acceleration. This cross section can be clearly predetermined on the basis of Figure 7a-d (right). Acceleration profiles captured at 3 times were plotted as a function of a normalized Lagrangian coordinate $X_{1}^{\star}$. Whatever the studied material, we observed that the profiles clearly intersect at a single point corresponding to the cross section where the crack finally occurs. 

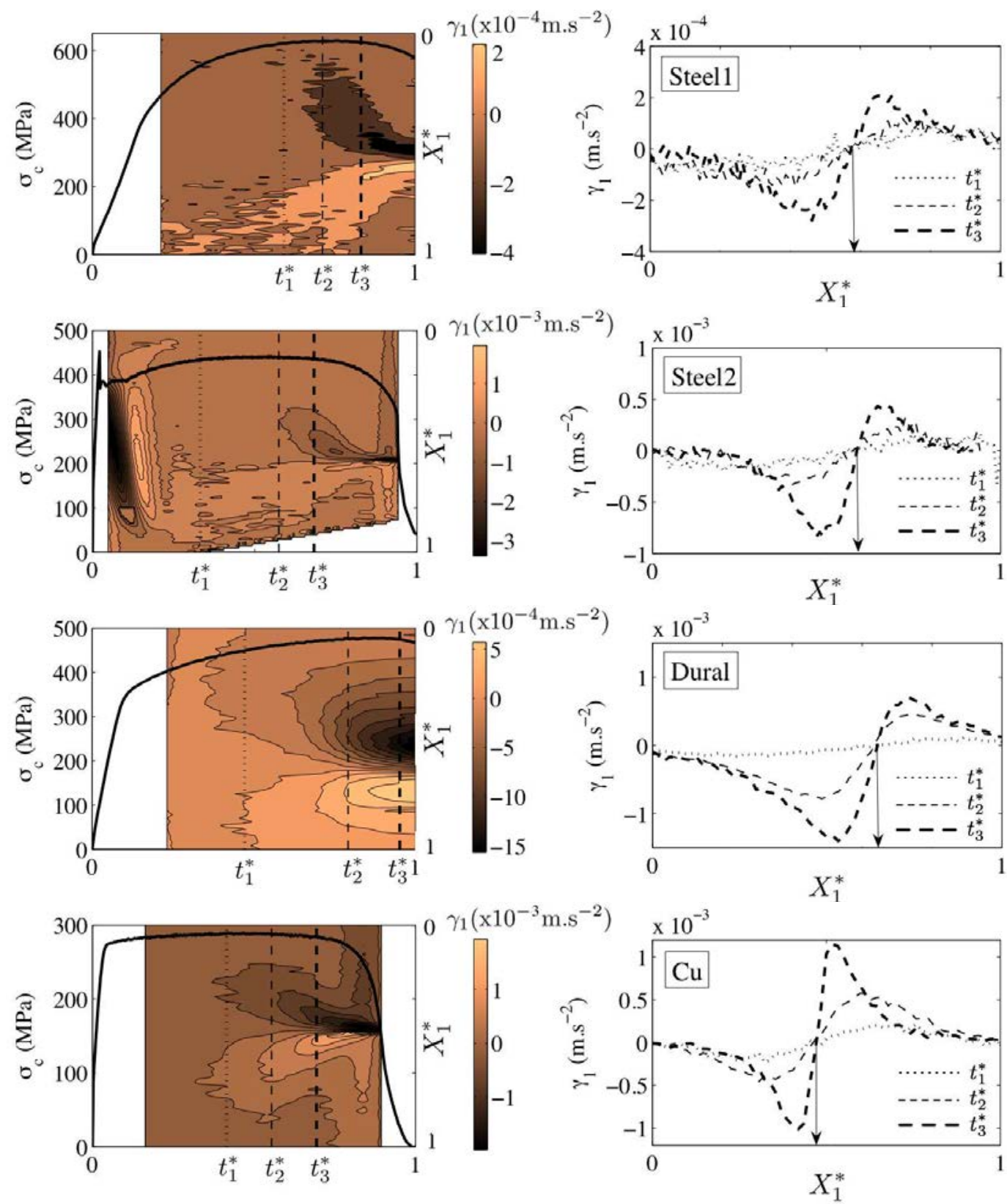

Figure 7: Longitudinal acceleration profiles: (a) Steel 1; (b) Steel 2; (c) Dural; (d) Cu. (left) Time course of the conventional stress (solid line) and spatiotemporal representation of accelerations (contour plots); (right) selected acceleration profiles at 3 different instants $\left(t_{1}^{\star}<t_{2}^{\star}<t_{3}^{\star}\right)$ marked by vertical lines on the left plots. 
The coordinate of this cross section did not systematically correspond to the centre $\left(X_{1}=L_{0} / 2\right)$ of the gauge length despite the considerable care exercised concerning accurate specimen polishing and positioning. This result is however not surprising if we think about the heterogeneous distribution of microstructural defects within the sample gauge part that may rapidly give rise to heterogeneous mesocopic responses of the material.

\subsection{Material rotations}

A 1D analysis of CZM using non-homogeneous tensile tests remains consistent as long as irrotational transformation is observed. Using the full-field displacement fields, maps of material rotations can be computed from the polar decomposition of the transformation gradient tensor $F=R U$, where $R$ is an orthogonal tensor while $U$ is a symmetric definite positive tensor. In order to visualize these rotations, we plotted in Figure 8 the distribution over the specimen gauge part of angles $\alpha=\left|\cos ^{-1} R_{11}\right|$ computed at different times for $\mathrm{Cu}$ specimens. At the beginning of the test, the greatest rotation angles occurred in the connection zone of the specimen where slight shear stresses developed (Figure 8 (left)). When necking occurred, the greatest rotations appeared on both sides of the necking shoulders (Figure 8 (middle)). However, the rotation angles $\alpha$ were less than 0.25 degree $\left(\simeq 5 \cdot 10^{-3} \mathrm{rad}\right.$. $)$ and remained negligible (Figure 8 (right)). Until the crack occurred, the maximal logarithmic shear strain $\varepsilon_{12}$, which was inside the specimen gauge part for the 4 materials, remained much lower than $10^{-2} \times \varepsilon_{11}$ throughout the test, with $\varepsilon_{11}$ corresponding to the associated tensile strain. The eigen directions of the strain tensor then remained fixed and parallel to directions $i=1,2,3$ already defined. 

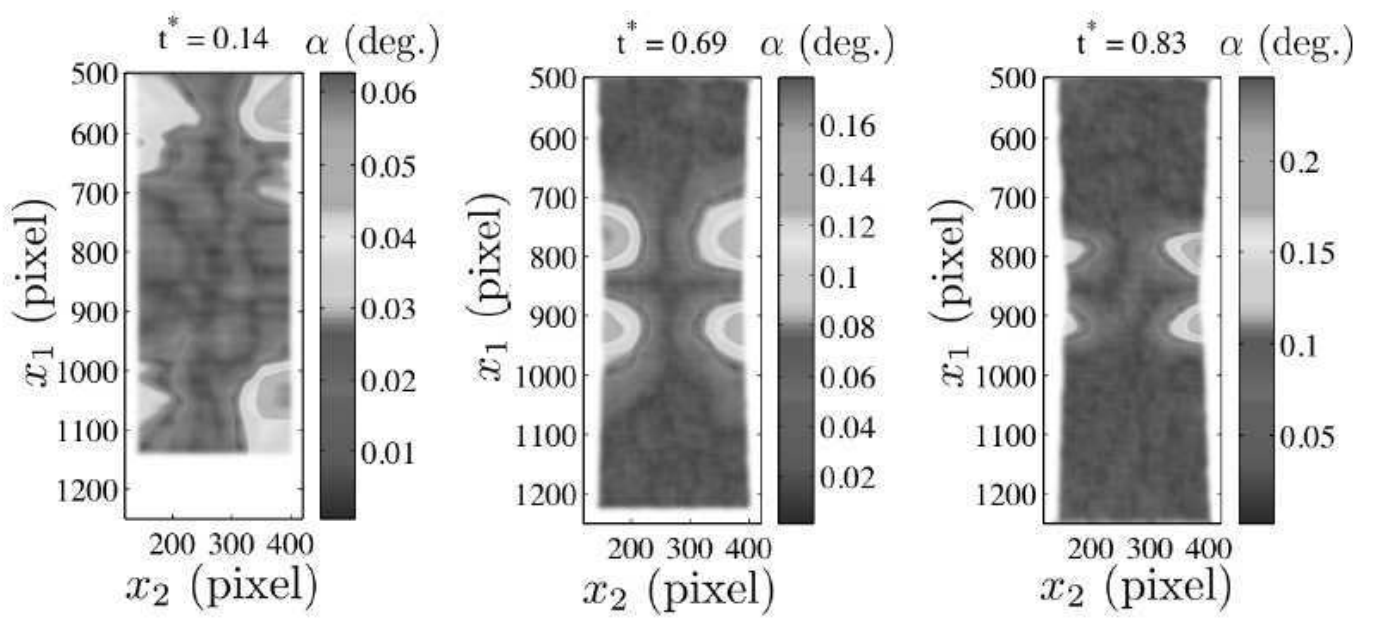

Figure 8: Material rotations (degrees) at 3 different times for $\mathrm{Cu}$ specimen.

The simple 1D mechanical analysis of the mechanical behavior presented hereafter can then be legitimated by the absence of significant rotations and the lowness of shear strains. In particular, an additive partition of elastoplastic strain is possible for a classical multiplicative decomposition of the transformation gradient (Lee, 1969). Indeed, with $F=F_{e} F_{p}$, it arises when there are no longer any rotations:

$$
\begin{aligned}
F & \simeq U=U_{e} U_{p} \\
\varepsilon & \simeq \log U=\log U_{e}+\log U_{p}=\varepsilon_{e}+\varepsilon_{p},
\end{aligned}
$$

where $U_{e}$ and $U_{p}$ are elastic and plastic dilatation tensors respectively associated with the polar decomposition of $F_{e}$ and $F_{p}$.

\subsection{Damage field}

Plasticity mechanisms are generally supposed to be isochoric. With elastic strain remaining small, volume variations are then associated with porosity induced by microvoids or opening of microcracks (Lemaitre, 1992). The 
kinematical data were then used to estimate volume variations associated with micro-void development and then to quantify the material volumetric damage. This measurement is the first step in identifying an 'equivalent surface damage' corresponding to a cohesive law.

By construction, the displacementtransformation gradient $F$ ef the transformation-is such that:

$$
\operatorname{det} F=\frac{\mathrm{d} v}{\mathrm{~d} V} \geq 0
$$

where $\mathrm{d} v$ and $\mathrm{d} V$ are respectively volumes occupied by an infinitesimal amount of matter in the current and reference configurations. The volume dilatation can be classically related to the Hencky strain tensor by:

$$
\frac{\mathrm{d} v}{\mathrm{~d} V}=e^{\operatorname{tr} \varepsilon}
$$

where operator 'tr' symbolizes the trace operator. Standard DIC technique gives us access only to in-plane components $u_{1}$ and $u_{2}$ of the displacement and consequently to $\varepsilon_{1}$ and $\varepsilon_{2}$. The out-of-plane component $u_{3}$ is not available. Nevertheless, analysis of the cross-section shape close to the necking regions led us to assume in a first approximation that $\varepsilon_{2}=\varepsilon_{3}$. For example, the isotropic contraction of cross sections has been quite well verified experimentally for the grade of steel shown in Wattrisse et al. (2001a). With such a transverse isotropy assumption, the local relative volume variation $\mathrm{d} v / \mathrm{d} V-1$ can be expressed only with the strain assessments:

$$
\frac{\mathrm{d} v}{\mathrm{~d} V}=e^{\left(\varepsilon_{1}+2 \varepsilon_{2}\right)},
$$

Since a pure plastic transformation of the material (i.e. excluding microvoids and the very slight elastic dilatation) remains isochoric, the relative 
volume variation can be regarded as a microvoid fraction within $\mathrm{d} V$, with elastic effects being negligible even at finite strain:

$$
\frac{\mathrm{d} v}{\mathrm{~d} V}-1=\frac{\mathrm{d} v_{v}}{\mathrm{~d} V}
$$

where $\mathrm{d} v_{v}$ is the microvoid volume. Since these microvoids reduce the surface that could withstand stress, a local scalar variable can be classically introduced to describe isotropic damage. This variable represents the surface proportion of microvoids (Chaboche, 1988; Ju, 1989; Kachanov, 1958, 1980; Krajcinovic, 1989; Lemaitre, 1992):

$$
D=\frac{\mathrm{d} s_{v}}{\mathrm{~d} S}
$$

where $\mathrm{d} s_{v}$ is, in this work, the surface of microvoids associated with the elementary surface $\mathrm{d} S$ perpendicular to the straining direction. Geometric modeling of microvoids is required to calculate the value of $D$ from volume changes. Different models already exist in the literature to take the damage by cavitation, decohesion of matrix particles, crazing, etc., into account. The main damage mechanisms concerning the metals under investigation are microcracks opening and cavitation. Following Wu et al. (2011) and for the sake of simplicity, we propose to assume a simple spherical void shape, distribution and growing: ( $i$ ) a uniform microvoid distribution, $(i i)$ with voids of the same spherical shape (radius $a$ ), (iii) uniformly distributed within a volume element, $(i v)$ and of same isotropic growth kinetics. The void density per unit length $\eta$ is thus the same for all directions. Considering a virgin specimen in the reference configuration, we get for an elementary cubic volume:

$$
\frac{\mathrm{d} v_{v}}{\mathrm{~d} V}=\frac{4}{3} \pi(a \eta)^{3} .
$$


According to Eq. (7) and the geometric assumptions made on the microvoid network, the local estimate of the damage parameter is: $D=\pi(a \eta)^{2}$, and can be expressed as a function of the relative volume variations derived from the kinematic measurements:

$$
D=\alpha_{D}\left(\frac{\mathrm{d} v_{v}}{\mathrm{~d} V}\right)^{2 / 3},
$$

with $\alpha_{D}=(3 / 4)^{2 / 3} \pi^{1 / 3} \simeq 1.2$.

Figure 9 (left) shows the time course of the relative volume variation profile along the straining direction for the 4 studied materials. Figure 9 (right) shows the damage evolution for 3 cross sections: the first one is centered on the necking zone (bold line) while the two others were chosen on each side of the crack zone (dashed lines). As often observed at the scale of the sample gauge part, in all cases failure occurs when local damage parameter values are lower than 1 . This is partly due to the current limitations of the CCD camera (spatial and temporal resolutions) and to the image processing (space-time fitting necessary to filter noisy and discrete data before derivation). It is also due to the academic character of the chosen porosity model, which becomes unsound as soon as anisotropic growth and coalescence of microvoids occur. Fortunately, the assessments show small damage values at this level and surely have a slight influence on what happens next. Moreover, the amplitude of the damage parameter in the zero acceleration necking zone (bold line) is larger than away from this zone (dashed lines): again, the zero acceleration zone corresponds exactly to the cross section where the failure occurs. The inverse problem to obtain some volumetric informations from the surface measurements is a complex problem. A more accurate estimate of the local volume variations using full-3D measurements 

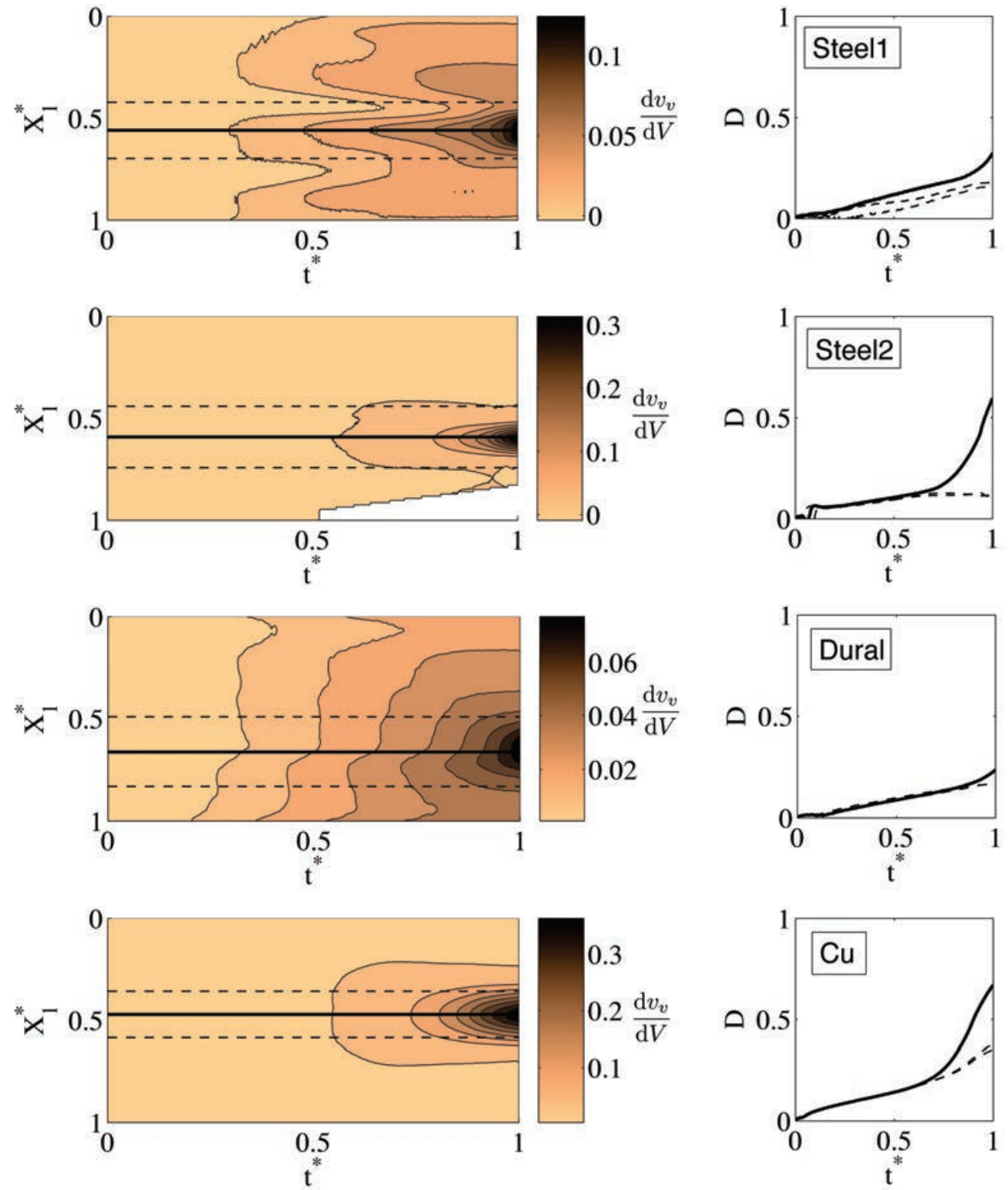

Figure 9: (Left) Time course representation of the Lagrangian longitudinal profile of the relative volume variations (isovalues). (Right) Kinetics of the damage parameter over 3 chosen cross sections located by the horizontal lines on the left figure. 
will be investigated in a future work. However, the recent work of Wu et al. (2011) concerning the damage measurement by full-3D DIC reports no value of damage greater than 0.4 and the validity of such type of approach could be probably challenged for very large strains. The damage maps identified with our 2D measurements are shown in Figure 10 before and during the strain localization: no particular spatial inhomogeneities can be pointed out in the direction perpendicular to the tensile direction. In the sequel, a pure 1D approach will thus be carried on.
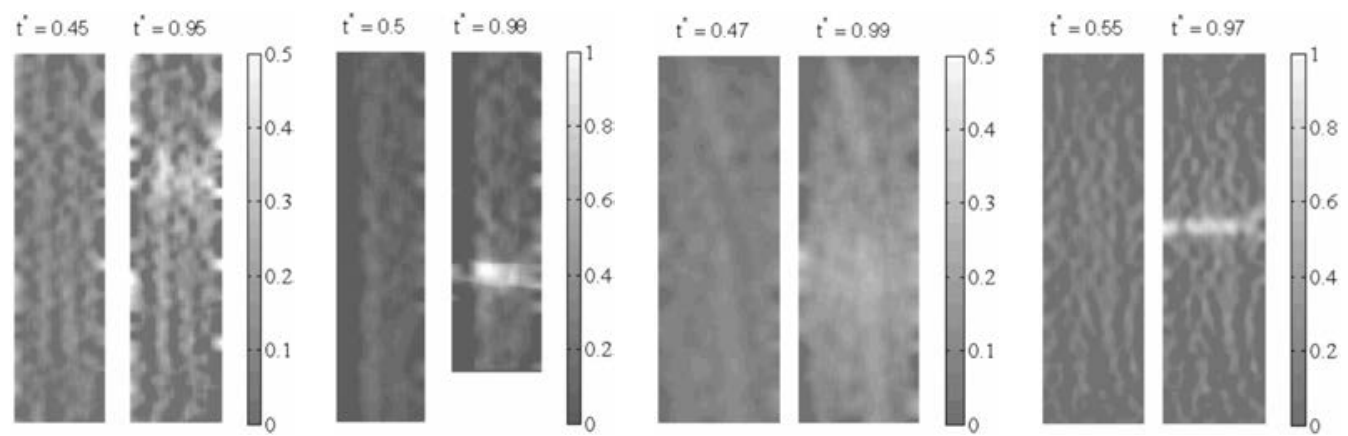

Figure 10: Damage maps before and during the strain localization (from left to right: Steel1, Steel2, Dural and Copper). Please note that the gray level bars were adapted to the range of the damage signal.

\subsection{Local stress-strain responses}

Since we have in mind to separate hardening bulk behaviors and softening surface ones, the local measurement of the damage field allows now to derive local stress-strain responses as well in the localization zone (where the crack will occur) as in the rest of the sample. 
For standard tensile tests, it is generally assumed that the tensile Cauchy stress is uniformly distributed over the cross section of the specimen. This tensile stress is classically written as

$$
\sigma\left(x_{1}, t\right)=\mathcal{F}(t) / S\left(x_{1}, t\right)
$$

where $S\left(x_{1}, t\right)$ is the current cross section of the specimen at the longitudinal Eulerian coordinate $x_{1}$ and time $t$. For $1 \mathrm{D}$ data processing, while taking the mass balance and the transverse isotropy hypothesis into account, $S\left(x_{1}, t\right)$ can be related to the initial cross section by:

$$
S\left(x_{1}, t\right)=S_{0} e^{2 \varepsilon_{2}\left(x_{1}, t\right)} .
$$

According to the definition of the damage parameter (Lemaitre, 1992), the stress $\sigma_{\text {eff }}$ relative to the effective surface is then:

$$
\sigma_{\text {eff }}\left(x_{1}, t\right)=\frac{\sigma\left(x_{1}, t\right)}{1-D\left(x_{1}, t\right)} .
$$

Figure 11 shows the stress-strain diagrams at different loci along the gauge part of the $\mathrm{Cu}$ specimen. The influence of the gradual development of heterogeneity on the local stress-strain response is clearly shown by their successive divergence. At the beginning of the test, all the responses follow the same path but do not move on this path at a same speed. The more the cross section is close to the localization zone, the longer is the covered path. Divergence of local stress-strain responses appears when the specimen softens. We have already discussed in Wattrisse et al. (2001b) the different possible interpretations of such softening in terms of material and/or structure effects. We particularly showed that strain softening properties strongly depend on the observation scale. In the framework of the present experiment, 

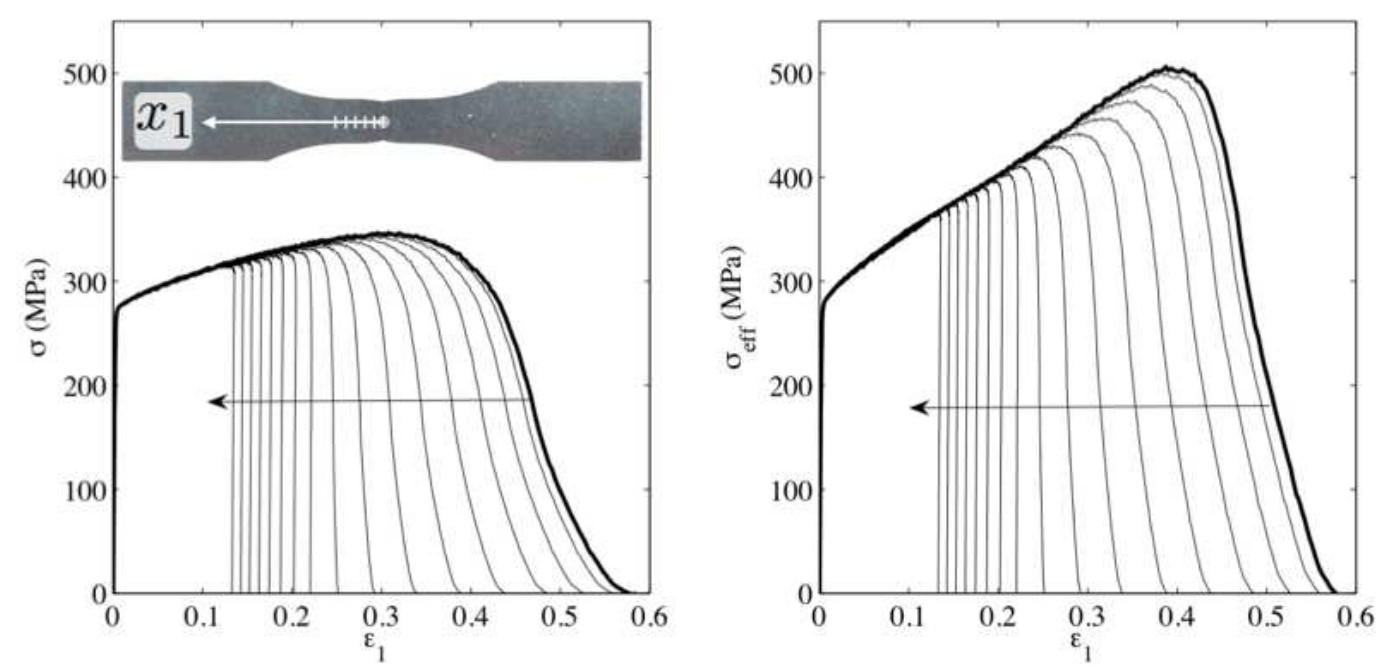

Figure 11: Local stress-strain diagrams $(\mathrm{Cu})$. (a) Cauchy stress vs. Hencky strain; (b) Effective stress vs. Hencky strain. The horizontal arrow indicates a growing distance from the localization zone (bold line).

softening of the specimen is associated with a local elastic unloading for cross sections placed outside the necking zone. For cross sections placed inside the current necking zone, the load decrease is accompanied by a local softening, as soon as the localization zone size becomes smaller than the gauge length fixed by the optical system used to estimate the local strain, as it is shown in Figure 11a-b. This scenario translatesdescribes the competition between the load decrease and the local necking course. It naturally changes from one cross-section to another. The horizontal arrow in Figure 11 symbolizes the increasing distance to the necking region of the cross section concerned by the local response curve. A comparison of Figure 11a and Figure 11b shows the influence of damage on the local stress-strain response. The envelope curve (bold line) corresponds to the material points located in the rupture cross section. This particular stress-strain curve is considered to identify the 
1D elastoplastic damageable response and extract the sought response of the cohesive zone.

\section{Towards a cohesive zone model identification}

Before using these experimental data, we propose to remind the objective of the CZM approach of fracture and to progressively introduce the identification protocol of cohesive zone laws using several academic examples.

\subsection{CZ response through heuristic $1 D$ models}

Whatever the damage mechanism, locations of defects (i.e. small regions where stresses are not transmitted) are not known a priori. In CZM-based simulations, failures only occur at predetermined locations, but a structure may also be weakened without being broken. This is reflected by the 'opening' of cohesive zones up to a value that corresponds to the maximum damage, therefore leading to structural failure. By construction, the CZM approach 'concentrates' the damage at the interfaces between adjacent volumetric elements (FE meshes or FE meshed bodies) and the numerical 'opening' does not represent a material displacement jump but instead summarizes at this interface the overall kinematical effects induced by the damage at the vicinity of this interface. From a strength standpoint now, CZM introduces cohesive forces associated with the displacement jump so that the work related to this couple represents the part of the volume deformation energy induced by the damage mechanisms. From a rheological standpoint, the introduction of cohesive zones leads to a clear separation of damage effects and implies a series construction of deformation mechanisms: one rheological component (the bulk part of the FE modeling) ranslatesdescribes the undamaged 
material behavior such as elastoplasticity with hardening, while a second component (the cohesive/surface part of the FE modeling) reflects the damage effects. The relevance and efficiency of this separation of damage effects are strongly related to the length scale at which the modeling is performed.

Usually, CZMs describe the material cohesion through a relationship between a cohesive stress $R_{C}$ and a displacement jump $[u]$ between two contiguous elements of the finite element mesh. From a more physical standpoint, the resulting jump must be interpreted as the overall displacement induced by damage (growth and coalescence of microvoids for example), and the onset and opening of a microcrack within a mesoscopic volume element. This displacement jump $[u]$ surely cannot be interpreted as the gap between the crack lips inasmuch as $R_{C}$ would consequently vanish for any $[u] \neq 0$. To construct the $R_{C}$ vs. $[u]$ relationship, the first step is to split the overall strain $\varepsilon$ into a bulk strain $\varepsilon_{B}$ that expresses the volumetric effects and a 'cohesive' strain $\varepsilon_{C}$ that summarizes the kinematic effects of damage effects. Hereafter this strain-formulated cohesive zone will simply be referred to as a cohesive zone.

The next subsection illustrates this additive strain decomposition through heuristic, analytical 1D models. These rather academic exercises led us to progressively construct a generic method to numerically perform this decomposition for a set of experimental data. 


\subsubsection{Damageable elasticity}

Let us consider the simple one-dimensional case of damageable elasticity. The constitutive equation classically reads:

$$
\sigma=(1-D) \sigma_{\text {eff }}=(1-D) E \varepsilon
$$

where $E$ is the Young modulus, and $D$ is a damage parameter. For the sake of simplicity, we avoided using any subscript for the 1D stress and strain component. We only considered tensile loading $(\sigma \geq 0)$. We also simply supposed that the damage rate is proportional to the positive part of the tensile strain rate expressed by:

$$
\dot{D}=\langle\dot{\varepsilon}\rangle^{+} / \varepsilon_{R} \quad \text { if } \quad \varepsilon=\varepsilon_{M},
$$

where $\varepsilon_{R}$ is the strain level at which rupture occurs, $\varepsilon_{M}=\max \{\varepsilon(\tau), \tau \leq t\}$ and $\langle x\rangle^{+}$symbolizes the positive part of $x$. The damage then increases when the strain reaches the yield strain $\varepsilon_{M}$ and as long as $\varepsilon_{M}$ is less than $\varepsilon_{R}$.

The time integration of Eq. (14) leads to:

$$
D(t)=\int_{0}^{t}\langle\dot{\varepsilon}(\tau)\rangle^{+} / \varepsilon_{R} \mathrm{~d} \tau=\frac{\varepsilon_{M}(t)}{\varepsilon_{R}} .
$$

As proposed above, let us now assume an additive decomposition of the overall strain $\varepsilon=\varepsilon_{B}+\varepsilon_{C}$ which allowed us to consider a series model, associating elasticity with a first component and damage with a second one We then formulate:

$$
\sigma=E \varepsilon_{B}=E\left(\varepsilon-\varepsilon_{C}\right)=R_{C}=h\left(\varepsilon_{C}\right),
$$

where $h$ is a function to be defined. 
For a monotone loading until rupture, Eqs. (13-16) lead to the following relation:

$$
\sigma=E\left\{1-\frac{1}{\varepsilon_{R}}\left(\frac{\sigma}{E}+\varepsilon_{C}\right)\right\}\left(\frac{\sigma}{E}+\varepsilon_{C}\right) .
$$

Solving Eq. (17) gives a single solution compatible with the physical boundary values $\sigma\left(\varepsilon_{C}=0\right)=0$ and $\sigma\left(\varepsilon_{C}=\varepsilon_{R}\right)=0$ and allows us to derive the function $h$ expression. The response of the strain-formulated 'cohesive zone' is:

$$
R_{C}=h\left(\varepsilon_{C}\right)=E\left(\sqrt{\varepsilon_{R} \varepsilon_{C}}-\varepsilon_{C}\right) .
$$

If unloading is considered at a given strain $\varepsilon=\varepsilon_{M}$, the damage stops at $D(t)=D_{M}=\varepsilon_{M} / \varepsilon_{R}$. Eqs. (13-16) allow us to derive the response of the cohesive zone during unloading:

$$
R_{C}=h\left(\varepsilon_{C}\right)=E\left(\frac{1-D_{M}}{D_{M}}\right) \varepsilon_{C} .
$$

The unloading response is then a linear function of the cohesive strain, with the slope characterizing the straight line of the $\sigma-\varepsilon_{C}$ plane passing through the origin and the maximum damage state reached at $\varepsilon=\varepsilon_{M}$. The slope tends to infinity for small damage values as the derivative $\mathrm{d} \sigma /\left.\mathrm{d} \varepsilon_{C}\right|_{\varepsilon_{C}=0}$ of the cohesive zone response during the loading.

If $\ell_{\mathrm{fe}}$ now represents a characteristic length associated with CZM, the cohesive law can then be written as:

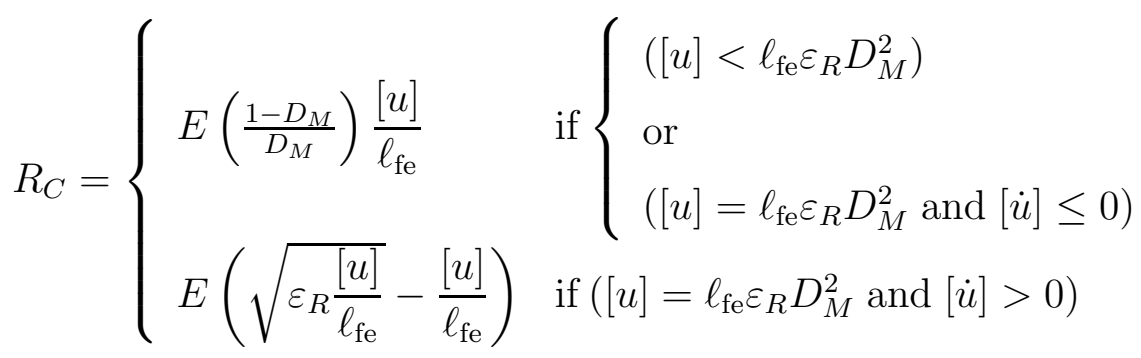


where $[u]=\ell_{\mathrm{fe}} \varepsilon_{C}$. Figure 12 illustrates the cohesive zone response associated with Eq. (20). Naturally, the validity of Eq. (20) is directly related to the consistency of the 'physics' included in the material constitutive Eqs. (13-14).

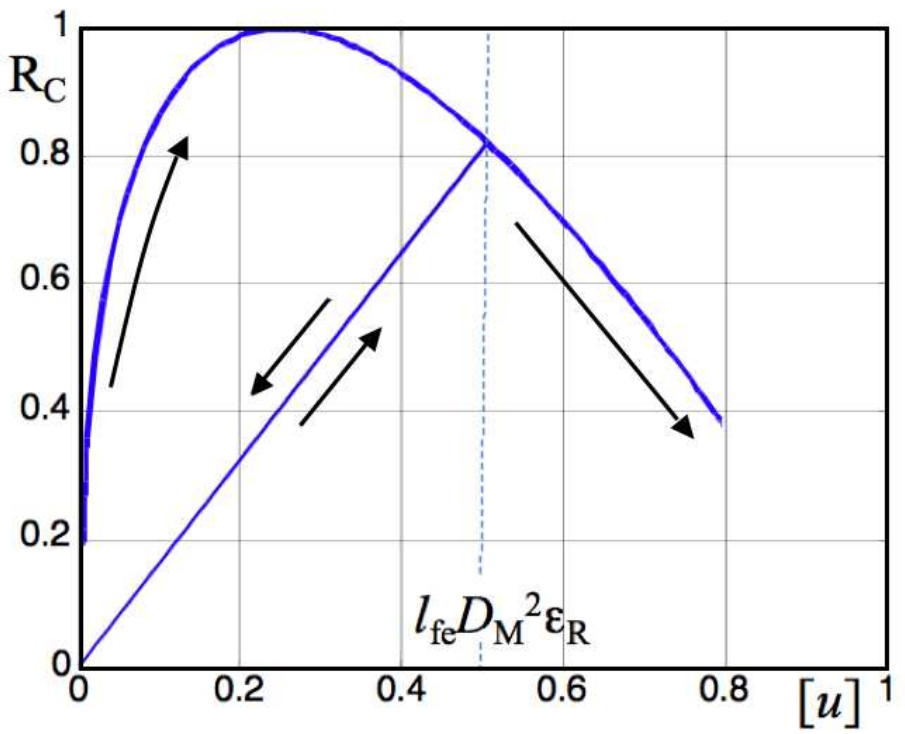

Figure 12: Example of normalized cohesive zone response for an elastic damageable material.

An extension of this damageable elasticity analysis to damageable elastoplastic materials is proposed in the next paragraph.

\subsubsection{Damageable elastoplastic model}

Analytical expression of the cohesive zone constitutive equation cannot be obtained systematically. In what follows, we present a generic method to extract the cohesive zone response in most complicated situations. The method is illustrated through the following damageable elastoplastic behavior. Once again, only tensile loadings $(\sigma \geq 0)$ will be considered. We admitted the principle of strain equivalence proposed by Chaboche and Lemaitre (1978). 

being introduced via the effective stress.

The elastic domain is defined by the yield function $f(\sigma, H)=\sigma-H-\sigma_{y} \leq$ 0 , where $\sigma_{y}$ is the elastic limit while $H$ stands for the power law hardening $H=K \varepsilon_{p}^{n}$, with $K$ being the so-called hardening modulus and $n$ the plastic exponent. The constitutive equations can be summarized by the following state and rate equations (with effective stress replacing the stress in the yield function $f$ ):

$$
\left\{\begin{array}{l}
\text { State equation: } \\
\sigma_{\text {eff }}=E\left(\varepsilon-\varepsilon_{p}\right) \\
\text { Evolution equation: } \\
\dot{\varepsilon_{p}}= \begin{cases}0 & \text { if } f<0 \text { or }(f=0 \text { and } \dot{f}<0) \\
\left(\frac{H}{K}\right)^{\frac{1-n}{n}} \frac{1}{n K} \dot{\sigma}_{\text {eff }} & \text { if } f=0 \text { and } \dot{f}=0\end{cases}
\end{array}\right.
$$

The damage time course is, once more, modeled by a simplistic function of strain:

$$
\dot{D}=\left\{\begin{array}{lll}
0 & \text { if } & \varepsilon<\varepsilon_{M} \\
m\left\langle\frac{\dot{\varepsilon}}{\varepsilon_{R}-\varepsilon_{0}}\right\rangle^{+}\left(\frac{\varepsilon-\varepsilon_{0}}{\varepsilon_{R}-\varepsilon_{0}}\right)^{m-1} & \text { if } & \varepsilon=\varepsilon_{M}
\end{array},\right.
$$

For monotone tests, combining equations (21), (22) and the additive partition $\varepsilon=\varepsilon_{B}+\varepsilon_{C}$ associated with the hypothesis of a series model, the stress 
reads:

$$
\sigma=(1-D) \sigma_{\mathrm{eff}}=g\left(\varepsilon_{B}\right)=g\left(\varepsilon-\varepsilon_{C}\right)=R_{C}=h\left(\varepsilon_{C}\right),
$$

where $g$ and $h$ are functions that respectively define the bulk and cohesive stress-strain responses ( $h$ does not have same expression as in (16) since the overall behavior has changed). The chosen elastoplastic bulk response is, by construction, described by Eq. (21). Roughly speaking, this bulk response corresponds to the overall when no damage occurs.

The following procedure is proposed to identify the :Ajoutercohesive response $h\left(\varepsilon_{C}\right)$. During monotone loading, the overall uniaxial response $\sigma-\varepsilon$ is recorded, as an increasing-decreasing function $\sigma=w(\varepsilon)$ (see Figure 13). At each computational step $k$, it is then possible to determine $\varepsilon_{B}$ as the solution of a nonlinear equation that we formally write as:

$$
\varepsilon_{B}^{k}=\left(\varepsilon^{k}-\varepsilon_{C}^{k}\right)=g^{-1}\left(\sigma^{k}\right) .
$$

The non-regularity of the plastic behavior led us to develop a numerical computation of $g^{-1}$. However, in this academic example, the function $g$ is piecewise given for monotone loading. First (hardening part), until the peak stress value $\sigma^{\text {peak }}$, i.e. $0<\varepsilon<\varepsilon^{\text {peak }}$ with $\varepsilon^{\text {peak }}$ such that $g\left(\varepsilon^{\text {peak }}\right)=\sigma^{\text {peak }}, g$ is given by a time integration of $(21)$ and $g^{-1}$ appears as $\varepsilon_{B}^{k}=g^{-1}\left(\sigma^{k}\right)=\left(\left(\sigma^{k}-\sigma_{y}\right) / K\right)^{1 / n}+\sigma^{k} / E$. Second (softening part), i.e. $\varepsilon>\varepsilon^{\text {peak }}$, the function $g$ corresponds to an elastic discharge and $g^{-1}$ reads $\varepsilon_{B}^{k}=g^{-1}\left(\sigma^{k}\right)=\left(\sigma^{k}-\sigma^{\text {peak }}\right) / E+\varepsilon^{\text {peak }}$. With the couples The set $\left(\sigma^{k}, \varepsilon^{k}, \varepsilon_{B}^{k}\right)$ being known, the sought response $\vartheta^{k}=R_{C}^{k}=h\left(c_{C}^{k}\right)$ cohesive function $h$ is then immediately derived for each 
step $k$ as: $\sigma^{k}=h\left(\varepsilon_{C}^{k}\right)$. The cohesive strain reads $\varepsilon_{C}^{k}=\varepsilon^{k}-\varepsilon_{B}^{k}=$ $w^{-1}\left(\sigma^{k}\right)+g^{-1}\left(\sigma^{k}\right)$ and the cohesive function is defined formally by $h=\left[\left(w^{-1}+g^{-1}\right]^{-1}\right.$.
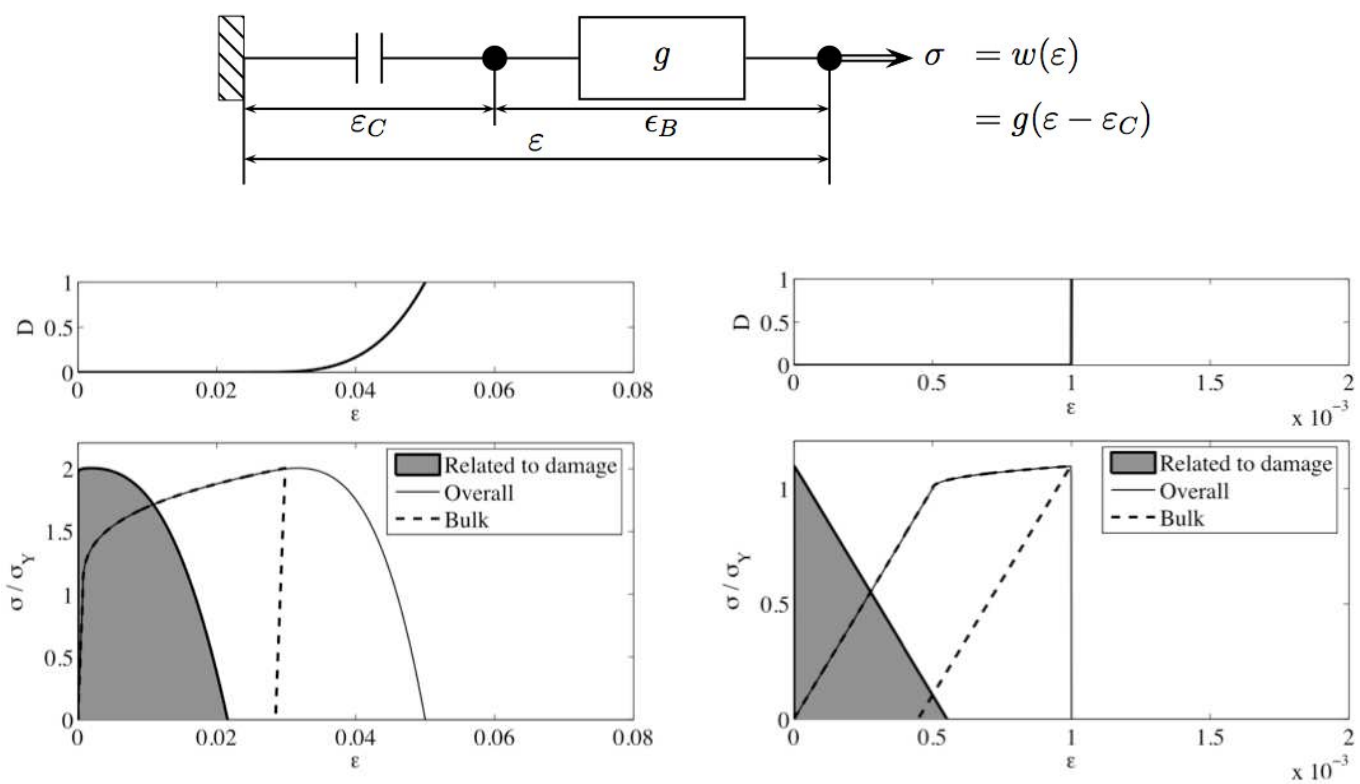

Figure 13: (Top) Series decomposition of a damageable elastoplastic response. Damage (Middle) and stress (Bottom) vs strain: (Left) Ductile material: $E=200 \mathrm{GPa}$, $\sigma_{y}=140 \mathrm{MPa}, \varepsilon_{0}=2.5 \%, \varepsilon_{R}=5 \%, K=440 \mathbf{G P a}, n=0.32, m=0.28$; (Right) Brittle material: $E=300 \mathrm{GPa}, \sigma_{y}=50 \mathrm{MPa}, \varepsilon_{0}=0.099 \%, \varepsilon_{R}=0.1 \%, K=400 \mathrm{MPa}$, $n=0.43, m=0.03$. The shaded curves correspond to the cohesive zone response $\left(\sigma / \sigma_{y}\right.$ versus $\left.\varepsilon_{C}\right)$.

Figure 13 shows the bulk response $g$ and that related to damage $h$ in the academic case of the damageable elasto-plastic model described above. Two sets of parameters corresponding to ductile and brittle behaviors were chosen. strong similarities with those of common (empirical) cohesive zone models described in the literature: trapezoidal shape (door-like) for ductile materials 
and linear shape (triangle) for brittle materials Alfano (2006); Borst (2002);

Chandra et al. (2002).

Note that the identified response $h\left(\varepsilon_{C}^{k}\right)$ is not a regular function since there is no initial compliance. The corresponding CZM (obtained by multiplying $\varepsilon_{C}$ by a characteristic length) is thus categorized as an 'extrinsic' model (i.e. with an infinite interfacial stiffness). In this case, this feature reflects the onset of damage from a stress threshold. The overall and bulk stress-strain relations are the same before any damage occurs.

This academic analysis shows how a damageable elastoplastic behavior can be locally splitted in the necking zone into a hardening volumetric behavior and a softening cohesive behavior. This approach is applied in the following to experimental measurements. Thus, in the sequel, the bulk function $g$ is not a priori given and will be implicitly deduced from the damage measurement: no assumption is made on the form of $g$ or on the damage time course. In other words, the experimental identification of the cohesive responses does not rest on simplistic state and rate relations (21) and (22) that were used in the previous academic example.

\subsection{Experimental analysis of cohesive zone responses}

When identifying a CZ model associated with an incompressible bulk behavior, the sample has to be considered as a structure in which there is a combination of structural and material effects. In this section, we propose a protocol to derive the uniaxial cohesive zone response associated with the loading direction during a monotonous tensile test. The objective is to determine the bulk part $\varepsilon_{B}$ and the elasto-plastic part $\varepsilon_{e}+\varepsilon_{p}$ from the measured 
tensile strain $\varepsilon_{1}$. The cohesive zone strain is, by construction, the difference:

$$
\varepsilon_{C}=\varepsilon_{1}-\varepsilon_{B}
$$

The chosen bulk behavior corresponds to isochoric elastoplasticity. To extract this underlying elastoplastic response from the experimental data, the compressibility induced by the damage development must be taken into account. The virtual equivalent stress $\sigma_{\text {inc }}$ associated with a given load $\mathcal{F}$ must verify:

$$
\mathcal{F}=\sigma_{\text {eff }} S_{\text {eff }}=\sigma_{\text {inc }} S_{\text {inc }}
$$

where $S_{\text {eff }}=S_{0}(1-D) e^{2 \varepsilon_{2}}$ and $S_{\text {inc }}=S_{0} e^{-\varepsilon_{1}}$, with the latter being associated with a virtual isochoric deformation process. During the experiments, the computation of $S_{\text {eff }}$ and $S_{\text {inc }}$ at each loading step required knowledge of the strain components and of the damage variable derived from Eqs. (5) and (9). Figure 14 presents a basic sketch of the additive partition of the strain where the isochoric stress is adjusted in accordance with Eq. (26).

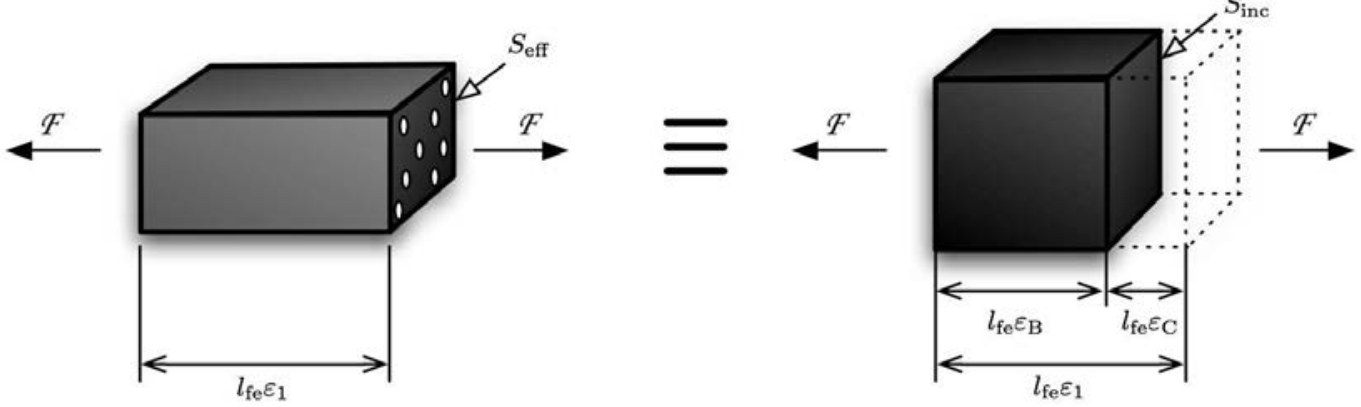

Figure 14: Basic sketch of the strain partition.

Regarding the effective behavior, the strain consists of an elastic and a plastic part:

$$
\varepsilon_{1}=\varepsilon_{e}+\varepsilon_{p}=\frac{\sigma_{\mathrm{eff}}}{E}+\varepsilon_{p},
$$


including the kinematical effects of the microvoids presence.

On the other hand, the isochoric elastoplasticity in series with a cohesive zone leads to:

$$
\varepsilon_{1}=\underbrace{\varepsilon_{e}^{\star}+\varepsilon_{p}^{\star}}_{\varepsilon_{B}}+\varepsilon_{C}=\frac{\sigma_{\text {inc }}}{E}+\varepsilon_{p}^{\star}+\varepsilon_{C} .
$$

Note that standard linear elasticity was assumed for effective and isochoric responses. This hypothesis remained closely in line with our observations during unloading.

As already mentioned, the cohesive zone response was determined during monotone loadings on the rupture cross section to ensure continuous progress of the plasticity until rupture (no elastic unloading). For such loading, $\sigma_{\text {eff }}$ represents the plastic flow stress as soon as $\sigma_{y}$ is reached. The flow stress can be formally written:

$$
\sigma_{\mathrm{eff}}=\sigma_{y}+H\left(\varepsilon_{p}\right)=\sigma_{y}+H\left(\varepsilon_{1}-\frac{\sigma_{\mathrm{eff}}}{E}\right)
$$

where $H$ characterizes the strain hardening. Young modulus $E$ and yield stress $\sigma_{y}$ are identified on the uniaxial and monotonic traction experiments. For any measured load $\mathcal{F}$, the principal components of the strain tensor, namely $\varepsilon_{1}$ and $\varepsilon_{2}$ (and $\varepsilon_{3}=\varepsilon_{2}$ assuming again transverse isotropy) are computed with the DIC procedure and appropriate spatial differentiations. Combining (5), (6) and (9), the corresponding damage is computed as $D \simeq 1.2\left(e^{\left(\varepsilon_{1}+2 \varepsilon_{2}\right)}-1\right)^{2 / 3}$. With (10), (11) and (12), the associated effective stress is estimated as $\sigma_{\text {eff }}=\mathcal{F} /\left(S_{0}(1-D) e^{2 \varepsilon_{2}}\right)$ in the actual configuration. These data together with (29) allow the numerical computation of the strain 
hardening function $H$ at any increment of elongation. In the same way, $\sigma_{\text {inc }}$ associated with $\sigma_{\text {eff }}$ (Eq. (26)) is the plastic flow stress developed by the incompressible bulk component. The principle of strain equivalence allows us to use the same hardening function $H$ to numerically compute the isochoric plastic strain as a root of the nonlinear equation $H\left(\varepsilon_{p}^{\star}\right)-\left(\sigma_{\mathrm{inc}}-\sigma_{y}\right)=0$. The cohesive strain can then be formally written:

$$
\varepsilon_{C}=\varepsilon_{1}-\underbrace{\frac{\sigma_{\mathrm{inc}}}{E}}_{\varepsilon_{e}^{\star}}-\underbrace{H^{-1}\left(\sigma_{\mathrm{inc}}-\sigma_{y}\right)}_{\varepsilon_{p}^{\star}} .
$$

Since the hardening response $H$ is experimentally known, finding $\varepsilon_{C}$ for a given value of $\sigma_{\text {inc }}$ leads to solve a non linear equation. In other words, the procedure is identical to the academic case described in section 4.1.2 with the bulk function $g$ (not a priori known in that case) now deduced from the overall response and the identification of the damage $D$.

Figure 15 shows, for the four studied specimens, the apparent stress $\sigma$ in the localization zone as a function of the overall and cohesive zone strains, respectively $\varepsilon_{1}$ and $\varepsilon_{C}$. The stress-strain relation $\sigma_{\text {inc }}$ vs. $\varepsilon_{B}$ associated with the virtual isochoric straining is also plotted on the same graphs. This relation corresponds to the bulk behavior that should be resolved at the Gauss points in a FE method for each material, and therefore it should be used for determining the bulk parameters of constitutive equations. The relation $\sigma$ vs. $\varepsilon_{C}$ provides access to the cohesive zone response and corresponds to the CZM, which can be directly used in CZM-based simulations through incorporation of the length $\ell_{\mathrm{fe}}$ corresponding to the link between the displacement jump $[u]$ and the cohesive strain $\varepsilon_{C}$, as mentioned in Section 4(20). The Figure 15 can 

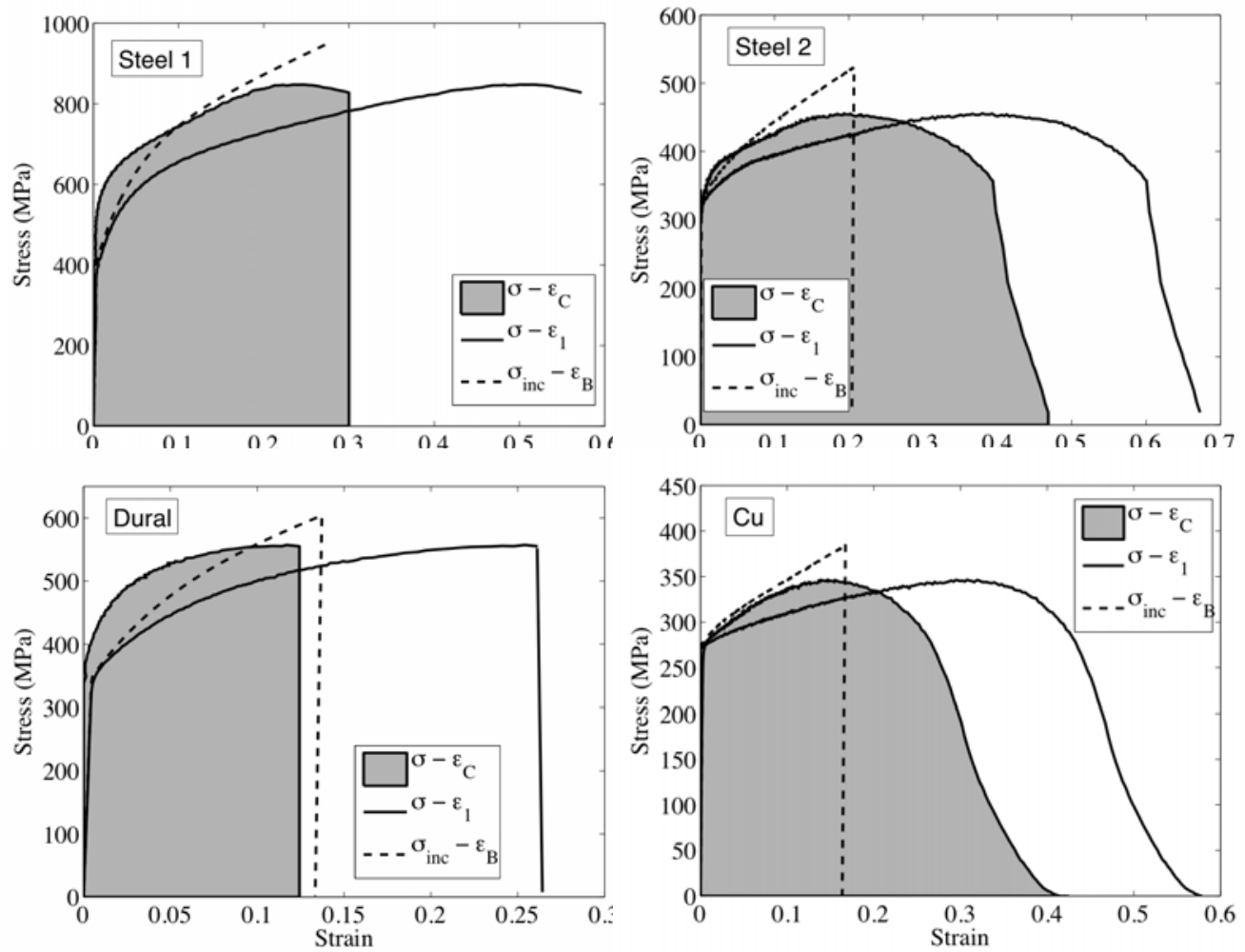

Figure 15: Local (within the localization zone) stress-strain diagrams for the studied materials. Measured $\sigma$ vs. $\varepsilon_{11}$ relation (solid line); Identified $\sigma_{\text {inc }}$ vs. $\varepsilon_{C}$ relation (dashed line); Identified cohesive zone response (solid line with grey area). 
be considered as the central result of this study: the cohesive-volumetric decomposition arises naturally from the uniaxial stress-strain response. While the apparent behavior (dark lines) is elastoplastic and damageable, the local bulk behavior (dashed lines) is elastoplastic, i.e. without any softening behavior, and the entire softening process is incorporated in the cohesive response (dark line with gray area). The local bulk responses exhibit higher hardening than the apparent responses and the maximal cohesive stress corresponds to the ultimate tensile stress of the apparent local behavior. Since the cohesive strain tends to increase only when the overall strain is high enough to induce some damage, the difference between the cohesive strain and the apparent strain at failure corresponds to the elastoplastic strain that the material can tolerate without any onset of microdamage.

This experimental protocol thus validates the cohesive-volumetric decomposition. However, at this stage of the study the final traction-separation relationship of the cohesive law cannot be obtained since the cohesive strain $\varepsilon_{C}$ has to be related to the displacement jump $[u]$ identifying the cohesive length $\ell_{\mathrm{fe}}$. The exact physical or numerical meaning of this length $\ell_{\mathrm{fe}}$ will be investigated in a forthcoming work. This length has indeed nothing to do with the length-scale used for the identification which is related to the CCD sensor characteristics, the optical lens, and the image processing. The relevance of CZM-based computational predictions depends, as usual, $(i)$ on the mechanical relevance of the chosen constitutive equation, $(i i)$ on the quality of the model identification and (iii) on the finenessrefinement of the discretization chosen in association with the estimated damage distribution heterogeneity level. 
In summary, the main elementary bricks of the proposed protocol are the following:

1. Use of the early character of the zone where the crack will finally occur to monitor the damage kinetics

2. Assessment of the local volume change distribution during the test using digital image correlation techniques

3. Correlation of this volume change to surface damage using a micromechanical model

4. Cohesive-volumetric decomposition using a dedicated strain partitioning.

Furthermore, we underline the fact that the second point has to be experimentally improved using, for example, stereo-correlations to avoid the transverse isotropy assumption, and that the third point can be replaced by any convenient micromechanical model or energy balance. However, the final result on the cohesive-volumetric decomposition is obtained here without loss of generality.

\section{Concluding comments}

In this paper, an experimental analysis on standard traction tests was proposed in order to identify cohesive zone models without any assumption on the shape of the cohesive law nor on the final crack path (like in debond tests). This experimental analysis allowed us to better grasp the physical sense of the so-called cohesive or interface law and displacement jump. We proposed an experimental approach to estimate 
local relative volume variations that correspond to microvoid fraction in the case of elastoplasticity (with negligible elastic volume changes). The damage parameter associated with these microvoids could be found through a voids growth model. Although the model used in this paper is very simple, it may be implemented to draw up an identification protocol of cohesive zone models using kinematical full-field measurements.

We identified cohesive zone responses for four different ductile materials and noted numerous similarities with the cohesive zone models commonly used in cohesive-volumetric finite element simulations. The three main results of this work are the following:

- a cohesive zone model can be experimentally identified for each given bulk behavior,

- such cohesive zone models belong to the class of so-called 'extrinsic' CZM,

- the classical empirical choices of door-like CZM for ductile materials and bilinear CZM for brittle materials are experimentally and theoretically confirmed.

In the extension of this work, it will be essential to perform numerical validations by comparing numerical and experimental results for different structures with complex loading paths in mode I. Pure shear tests should now be considered to complete the identification of cohesive zone models. It would also be of interest to extend the identification method to the brittle behavior corresponding to a wide range of materials in dynamic loading 
conditions. Moreover, the proposed strategy for CZM identification will be improved in a forthcoming work: 1/ the accuracy of the 2D approach has to be checked by complementary $3 \mathrm{D}$ measurements, 2/ the assumption of an underlying volumetric damage model has to be further analyzed (for example with the help of loading-unloading experiments), 3 / the cohesive length — as the key-parameter of CZM — has to be identified by comparison with numerical simulations.

\section{Acknowledgments}

This work was accomplished in the MIST Laboratory (joined Laboratory IRSN, CNRS and Université Montpellier 2, France). We would like to thank IRSN (French Institut de Radioprotection et de Sûreté Nucléaire) for providing financial support for this work.

\section{References}

Alfano, G., 2006. On the influence of shape of the interface law on the application of cohesive-zone models. Composites Science and Technology 66, $723-730$

Andena, L., Rink, M., Williams, J. G., 2006. Cohesive zone modelling of fracture in polybutene. Engineering Fracture Mechanics 73 (16), 24762485.

Arias, I., Knap, J., Chalivendra, V. B., Hong, S., Ortiz, M., Rosakis, A. J., 2007. Numerical modelling and experimental validation of dynamic frac- 
ture events along weak planes. Computer Methods in Applied Mechanics and Engineering 196 (37-40 SPEC. ISS.), 3833-3840.

Barenblatt, G., 1962. The mathematical theory of equilibrium of cracks in brittle fracture. Adv. Appl. Mech. 7, 55-129.

Bornert, M., Brémand, F., Doumalin, P., Dupré, J., Fazzini, M., Grédiac, M., Hild, F., Mistou, S., Molimard, J., Orteu, J., Robert, L., Surrel, Y., Vacher, P., Wattrisse, B., 2009. Assessment of measurement errors in local displacements by digital image correlation: methodology and results. Experimental Mechanics 49 (3), 353-370.

Borst, R. D., 2002. Fracture in quasi-brittle materials: a review of continuum damage-based approaches. Engineering Fracture Mechanics 69, 95-112.

Chaboche, J., 1988. Continuum damage mechanics. Journal of applied mechanics 55, 59-71.

Chaboche, J., Feyel, F., Monerie, Y., 2001. Interface debonding models: a vicous regularization with a limited rate dependency. International Journal of Solids and Structures 38, 3127-3160.

Chaboche, J., Lemaitre, J., 1978. Aspects phénoménologiques de la rupture par endommagement. J. de Mécanique Appliquée 2 (3), 317-365.

Chandra, N., Li, H., Shet, C., Ghonem, H., 2002. Some issues in the application of cohesive zone models for metal-ceramic interfaces. International Journal of Solids and Structures 39, 2827-2855. 
Chrysochoos, A., Berthel, B., Latourte, F., Pagano, S., Wattrisse, B., Waber, B., 2008. Local energy approach to steel fatigue. Strain 44, 327-334.

Corr, D., Accardi, M., Graham-Brady, L., Shah, S., 2007. Digital image correlation analysis of interfacial debonding properties and fracture behavior in concrete. Engineering Fracture Mechanics 74 (1-2), 109-121.

Daly, S., Miller, A., Ravichandran, G., Bhattacharya, K., 2007. An experimental investigation of crack initiation in thin sheets of nitinol. Acta materialia 55 (18), 6322-6330.

de Borst, R., Remmers, J., Needleman, A., 2006. Mesh-independent discrete numerical representations of cohesive-zone models. Engineering Fracture Mechanics 73 (2), 160-177.

Dugdale, D., 1960. Yielding of steel sheets containing slits. Journal of the Mechanics and Physics of Solids 8, 100-104.

Fang, Q., Wang, T., Li, H., 2006. Large tensile deformation behavior of pc/abs alloy. Polymer 47 (14), 5174-5181.

Fedele, R., Raka, B., Hild, F., Roux, S., 2009. Identification of adhesive properties in glare assemblies using digital image correlation. Journal of the Mechanics and Physics of Solids 57 (7), 1003-1016.

Fuchs, P., Major, Z., 2011. Experimental determination of cohesive zone models for epoxy composites. Experimental Mechanics 51 (5), 779-786.

Hong, S., Kim, K., 2003. Extraction of cohesive-zone laws from elastic far- 
fields of a cohesive crack tip: a field projection method. Journal of the Mechanics and Physics of Solids 51, 1267-1286.

Huon, V., Wattrisse, B., Youssoufi, M. E., Chrysochoos, A., 2007. Elastic behavior of anisotropic terra cotta ceramics determined by kinematic fullfield measurements. Journal of the European Ceramic Society 27 (5), 23032310.

Jiang, L. Y., 2010. A cohesive law for carbon nanotube/polymer interface accounting for chemical covalent bonds. Mathematics and Mechanics of Solids 15 (7), 718-732.

Ju, J., 1989. On energy-based coupled elastoplastic damage theories: constitutive modelling and computational aspects. International Journal of Solids and Structures 25 (7), 803-833.

Kachanov, L., 1958. On the time to failure under creep conditions. IzV. AN SSSR, Otd. Tekhn Nauk 8, 26-31.

Kachanov, M., 1980. Continuum model of medium with cracks. ASCE J. Eng. Mech. Div. 106 (EM5), 1039-1051.

Krajcinovic, D., 1989. Damage mechanics. Mech. Mater. 8, 117-197.

Kubair, D., Geubelle, P., 2003. Comparative analysis of extrinsic and intrinsic cohesive models of dynamic fracture. International Journal of Solids and Structures 40 (15), 3853-3868.

Latourte, F., Chrysochoos, A., Pagano, S., Wattrisse, B., 2008. Elastoplastic 
behavior identification for heterogeneous loadings and materials. Experimental Mechanics 48 (4), 435-449.

Lee, E., 1969. Elastic-plastic deformation at finite strains. ASME J. Appl. Mech. 36, 1-6.

Lemaitre, J., 1992. A course on damage mechanics. Springer-Verlag Ed.

Lopez-Crespo, P., Shterenlikht, A., Yates, J., Patterson, E., Withers, P., 2009. Some experimental observations on crack closure and crack-tip plasticity. Fatigue and Fracture of Engineering Materials and Structures 32, $418-429$

Mekky, W., Nicholson, P., 2006. Bridging-stress evaluation in a Ni/Al2O3 system via the digital image-correlation technique. American Ceramic Society 89 (3), 1095-1098.

Ngo, D., Park, K., Paulino, G. H., Huang, Y., 2010. On the constitutive relation of materials with microstructure using a potential-based cohesive model for interface interaction. Engineering Fracture Mechanics 77 (7), $1153-1174$.

Perales, F., Dubois, F., Monerie, Y., Piar, B., Stainier, L., 2010. Multibody nscd strategy as a multi-domain solver. application to code coupling dedicated to the modeling of fracture of heterogeneous media. European Journal of Computational Mechanics 19, 189-417.

Shen, B., Paulino, G., 2011. Direct extraction of cohesive fracture properties from digital image correlation: A hybrid inverse technique. Experimental Mechanics 51 (2), 143-163. 
Tan, H., Liu, C., Huang, Y., Geubelle, P., 2005. The cohesive law for particle/matrix interfaces in high explosives. Journal of the Mechanics and Physics of Solids 53 (8), 1892-1917.

Tomar, V., Zhai, J., Zhou, M., 2004. Bounds for element size in a variable stiffness cohesive finite element model. International Journal for Numerical Methods in Engineering 61 (11), 1894-1920.

Tvergaard, V., Hutchinson, J., 1992. The relation between crack growth resistance and fracture process parameters in elastic-plastic solids. Journal of the Mechanics and Physics of Solids 40, 1377-1397.

Valoroso, N., Fedele, R., 2010. Characterization of a cohesive-zone model describing damage and de-cohesion at bonded interfaces. sensitivity analysis and mode-i parameter identification. International Journal of Solids and Structures 47 (13), 1666-1677.

Wattrisse, B., Chrysochoos, A., Muracciole, J., Némoz-Gaillard, M., 2001a. Analysis of strain localization during tensile tests by digital image correlation. Experimental Mechanics 41, 29-39.

Wattrisse, B., Chrysochoos, A., Muracciole, J., Némoz-Gaillard, M., 2001 b. Kinematic manifestations of localisation phenomena in steels by digital image correlation. European Journal of Mechanics - A/Solids 20 (2), 189211.

Wattrisse, B., Muracciole, J., Chrysochoos, A., 2001c. Thermomechanical effects accompanying the localized necking of semi-crystalline polymers. Int. J. of Therm. Sci. 41, 422-427. 
${ }_{737} \mathrm{Wu}$, T., Coret, M., Combescure, A., 2011. Strain localization and damage 738 measurement by full 3D digital image correlation: application to $15-5 \mathrm{PH}$ $739 \quad$ stainless steal. Strain 47, 49-61.

Zhu, Y., Liechti, K., Ravi-Chandar, K., 2009. Direct extraction of ratedependent traction-separation laws for polyurea/steel interfaces. Interna742 tional Journal of Solids and Structures 46 (1), 31-51. 